\title{
Ice hydrometeor profile retrieval algorithm for high-frequency microwave radiometers: application to the CoSSIR instrument during TC4
}

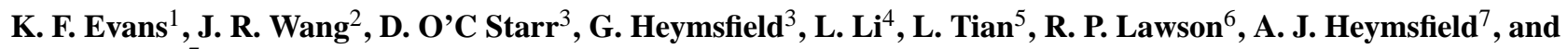 \\ A. Bansemer ${ }^{7}$ \\ ${ }^{1}$ Department of Atmospheric and Oceanic Sciences, University of Colorado, Boulder, CO, USA \\ ${ }^{2}$ Science Systems and Application, Inc., Lanham, MD, USA \\ ${ }^{3}$ Mesoscale Atmospheric Processes Branch, NASA Goddard Space Flight Center, Greenbelt, MD, USA \\ ${ }^{4}$ Microwave Instrument and Technology Branch, NASA Goddard Space Flight Center, Greenbelt, MD, USA \\ ${ }^{5}$ GESTAR, Morgan State University, NASA Goddard Space Flight Center, Greenbelt, MD, USA \\ ${ }^{6}$ SPEC, Inc., Boulder, CO, USA \\ ${ }^{7}$ National Center for Atmospheric Research, Boulder, CO, USA \\ Correspondence to: K. F. Evans (evans@ nit.colorado.edu)
}

Received: 12 February 2012 - Published in Atmos. Meas. Tech. Discuss.: 27 April 2012

Revised: 18 August 2012 - Accepted: 2 September 2012 - Published: 25 September 2012

\begin{abstract}
A Bayesian algorithm to retrieve profiles of cloud ice water content (IWC), ice particle size $\left(D_{\text {me }}\right)$, and relative humidity from millimeter-wave/submillimeter-wave radiometers is presented. The first part of the algorithm prepares an a priori file with cumulative distribution functions (CDFs) and empirical orthogonal functions (EOFs) of profiles of temperature, relative humidity, three ice particle parameters (IWC, $D_{\mathrm{me}}$, distribution width), and two liquid cloud parameters. The a priori CDFs and EOFs are derived from CloudSat radar reflectivity profiles and associated ECMWF temperature and relative humidity profiles combined with three cloud microphysical probability distributions obtained from in situ cloud probes. The second part of the algorithm uses the CDF/EOF file to perform a Bayesian retrieval with a hybrid technique that uses Monte Carlo integration (MCI) or, when too few MCI cases match the observations, uses optimization to maximize the posterior probability function. The very computationally intensive Markov chain Monte Carlo (MCMC) method also may be chosen as a solution method. The radiative transfer model assumes mixtures of several shapes of randomly oriented ice particles, and here random aggregates of spheres, dendrites, and hexagonal plates are used for tropical convection. A new physical model of stochastic dendritic snowflake aggregation is de-
\end{abstract}

veloped. The retrieval algorithm is applied to data from the Compact Scanning Submillimeter-wave Imaging Radiometer (CoSSIR) flown on the ER-2 aircraft during the Tropical Composition, Cloud and Climate Coupling (TC4) experiment in 2007. Example retrievals with error bars are shown for nadir profiles of IWC, $D_{\text {me }}$, and relative humidity, and nadir and conical scan swath retrievals of ice water path and average $D_{\text {me }}$. The ice cloud retrievals are evaluated by retrieving integrated $94 \mathrm{GHz}$ backscattering from CoSSIR for comparison with the Cloud Radar System (CRS) flown on the same aircraft. The rms difference in integrated backscattering is around $3 \mathrm{~dB}$ over a $30 \mathrm{~dB}$ range. A comparison of CoSSIR retrieved and CRS measured reflectivity shows that CoSSIR has the ability to retrieve low-resolution ice cloud profiles in the upper troposphere.

\section{Introduction}

There is ongoing interest in remote sensing of ice clouds due to their importance in radiative cloud feedbacks, precipitation, and upper troposphere water cycling. A fundamental ice cloud parameter is mass, such as ice water content (IWC) or its vertical integral, ice water path (IWP), which is needed 
to evaluate ice clouds in modern general circulation models. There are several ice cloud mass remote sensing techniques in use from satellites, including solar reflectance (e.g., Rossow and Schiffer, 1999; King et al., 1997), nadir viewing microwave (e.g., Ferraro et al., 2005), microwave limb sounding (e.g., Wu et al., 2006; Rydberg et al., 2009), and the CloudSat radar (Stephens et al., 2008; Austin et al., 2009). All of these approaches to sensing ice cloud mass apply to limited ranges of IWP, have limited spatial coverage, and/or have relatively low accuracy. In fact, comparisons of global ice mass datasets from these techniques (Wu et al., 2009; Eliasson et al., 2011) generally show poor agreement. Ice cloud mass can be obtained with higher accuracy from these satellite instruments using retrieval algorithms that combine instruments (e.g., Delanoe and Hogan, 2010).

High-frequency $(150 \mathrm{GHz}$ to $900 \mathrm{GHz}$ ) microwave (or submillimeter-wave) radiometry is a developing technique for remotely sensing ice cloud mass (Gasiewski, 1992; Evans and Stephens, 1995b; Evans et al., 1998, 2005; Buehler et al., 2007). Scanning submillimeter radiometry has some advantages over existing techniques in that it is fundamentally more sensitive to ice particle mass (and thus potentially has the best IWP accuracy) and has good spatial coverage from low Earth orbit.

Regardless of the technique, remote sensing ice cloud mass is difficult because there are many confounding factors that affect the measured radiances or backscattering. Depending on the technique, these factors include ice particle shape, particle size distribution, cloud height or temperature, vertical variability in the cloud, absorption by water vapor, attenuation by the cloud itself, effect of liquid cloud in and below the ice cloud, and surface emissivity or reflectivity. Some ice cloud sensing techniques have multiple wavelengths that give independent information to solve for some of these factors, but all techniques require a priori information about many of these factors. The simpler retrieval algorithms fix any factor that cannot be retrieved, for example, assuming a particular mixture of particle shapes, a fixed size distribution for each effective radius, homogeneous ice cloud, no underlying water clouds, and a specified surface albedo depending on surface type. Making these assumptions then allows forward radiative transfer modeling to be used to construct a lookup table that, for example, relates two observed radiances to water path and effective radius. These fixed assumptions might be fairly arbitrary or based on careful analysis of in situ ice cloud data and other a priori sources. More sophisticated retrieval algorithms deal with the fact that in the real atmosphere the confounding factors vary over certain ranges and covary with each other.

The usual underlying framework for this approach is Bayes' theorem and Bayesian probability concepts. In the Bayesian framework a priori information is specified with a probability density function (pdf). A Bayesian pdf specifies how likely the parameter is to have particular values. Thus, a Bayesian prior pdf should specify realistic distributions of parameter values and their inter-relationships before the measurements are applied. An important example for ice clouds is that we know from in situ measurements that characteristic particle size is negatively correlated with temperature and positively correlated with ice water content, and both IWC and particle size are positive and have a distribution that is much closer to log-normal than normal. Bayes' theorem says that the posterior pdf is proportional to the product of the prior pdf and the likelihood pdf, which is the conditional probability of the measurement vector given a particular atmospheric state. In the Bayesian framework the retrieved parameter, say IWP, is not a single value, but a whole posterior pdf specifying a range of likely values. It is difficult to deal with a retrieved pdf for each pixel, so usually the posterior pdf is summarized with its mean or mode and standard deviation (or other measure of its width). In cases of multiple modes in the posterior pdf, these summarizing quantities can be quite misleading.

One special case of a Bayesian retrieval methodology that has become popular recently in ice cloud remote sensing is optimal estimation, usually in the framework developed by Rodgers (2000). Examples of retrieval algorithms that use optimal estimation include the CloudSat IWC algorithm (Austin et al., 2009) and combined radar, lidar, and infrared radiometer algorithms (Zhang and Mace, 2006; Delanoe and Hogan, 2010). The optimal estimation framework of Rodgers (2000) has also been used to explore the information content of various visible and infrared wavelengths for retrieving ice clouds (Cooper et al., 2006). Optimal estimation is simpler and often more efficient than the fully general Bayesian framework because it assumes that the prior and likelihood pdfs are Gaussian and that the forward radiative transfer function is only moderately nonlinear. By transforming to $\log$ variables, optimal estimation can also be used easily with lognormal distributions as was done in Austin et al. (2009). Unfortunately, optimal estimation is sometimes poorly implemented in cloud remote sensing. The prior pdf covariance matrix is often assumed to be diagonal, ignoring the considerable information contained in the known correlations between variables. The prior pdf parameters are sometimes chosen somewhat arbitrarily, rather than being obtained from prior information contained in independent (e.g., in situ) datasets. Since optimal estimation uses Gauss-Newton iterations with the nonlinear forward model in the loop, there is a tendency to oversimplify the radiative transfer to speed the solution. Atmospheric parameters that ought to vary according to a prior pdf (because they affect the observations) are often fixed to simplify and speed the solution. For these reasons and because the forward function is not linear over the range of retrieval uncertainty, the retrieval errors from optimal estimation are usually substantially underestimated.

The more general Bayesian formulation has been used in some high-frequency microwave ice cloud retrieval algorithms, usually with a Monte Carlo integration approach (Evans et al., 2002, 2005; Seo and Liu, 2005; Rydberg et 
al., 2009). The Monte Carlo integration (MCI) method randomly generates atmosphere/cloud cases according to a prior probability density function and simulates instrument measurements for each case with a radiative transfer model to create a "retrieval database" of simulated observations and corresponding retrieval quantities. Since the cases in the retrieval database are already distributed according to the prior pdf, Monte Carlo integration over the Bayesian posterior distribution is performed by weighting the retrieved quantities in the database by the likelihood function. The likelihood function is usually assumed to be a Gaussian distribution, which is negligible unless the observation vector matches the simulated observation of the database case within the uncertainties. The standard deviation of the retrieved quantities weighted by the likelihood function can give uncertainty estimates.

The algorithm of Evans et al. (2002, 2005) generated retrieval databases having discrete ice cloud layers with cloud top altitude from a Gaussian distribution and cloud thickness from an exponential distribution. The microphysical properties at the top and bottom of an ice cloud were obtained stochastically from a pdf relating temperature, IWC, and median mass diameter derived from in situ cloud probe data. For each ice cloud, one of a few particle shapes was chosen randomly. Temperature and relative humidity profiles were generated stochastically using empirical orthogonal functions (EOFs) from statistics obtained from soundings. The Bayesian MCI method was used to retrieve IWP and median mass diameter from the observations.

Seo and Liu (2005) used EOF analysis to generalize ground-based radar reflectivity profiles and used Z-IWC relations to derive IWC profiles. Five different mixtures of six particle shapes and gamma distribution parameters were chosen stochastically. Temperature and humidity profiles were obtained by choosing from many radiosonde profiles. A database of $2.5 \times 10^{6}$ cases was thus generated and used to retrieve IWP from the five AMSU-B channels $(89,150$, $183.3 \pm 1, \pm 3, \pm 7 \mathrm{GHz})$.

Rydberg et al. (2009) generated three-dimensional (3-D) fields of ice cloud parameters using CloudSat radar data expanded to 3-D with a stochastic Fourier algorithm (Venema et al., 2006) and a fixed ice particle size distribution parameterization. Temperature and humidity profiles from ECMWF were stochastically modified to introduce small-scale variability. A retrieval database was generated by simulating radiances for the Odin-SMR limb-sounder at 501 and $544 \mathrm{GHz}$ from the 3-D fields, and Bayesian MCI was used to retrieve IWC and relative humidity profiles.

The MCI method uses a database and weighting by the likelihood function to perform Bayesian retrievals. A related technique uses an a priori retrieval database to train neural networks to retrieve ice cloud parameters from brightness temperature vectors. Examples of high-frequency microwave retrievals of IWP using this neural network method include Jimenez et al. (2007) and Defer et al. (2008). Defer et al.
(2008) used a cloud resolving model with several categories of ice particles to generate a database at frequencies from 24 to $875 \mathrm{GHz}$ to retrieve precipitation rate and IWP.

This paper describes a new Bayesian algorithm that retrieves ice cloud profiles and vertically integrated parameters. An overview of the retrieval algorithm is given in the next section. Sections 3 and 4 describe the algorithm in detail. Examples of retrievals with CoSSIR data and validation with CRS reflectivities are shown in Sect. 5. Section 6 discusses pros and cons of the retrieval algorithm, summarizes the results, and discusses future algorithm improvements.

\section{Overview of the retrieval algorithm}

The algorithm retrieves ice water content, ice particle size, and relative humidity profiles, and vertically integrated cloud parameters from microwave radiances and/or radar reflectivity profiles. The retrieval algorithm is tested with data from the Compact Scanning Submillimeter-wave Imaging Radiometer (CoSSIR, Evans et al., 2005) flown on the NASA ER-2 aircraft in July and August 2007 during the Tropical Composition, Cloud and Climate Coupling (TC4) experiment (Toon et al., 2010). CoSSIR measured brightness temperatures in 11 double sideband channels around 183.3, 220.0, 380.2, 640.0 and 873.6 GHz. Data are used, mostly for validation, from the nadir viewing $94 \mathrm{GHz}$ Cloud Radar System (CRS) (Li et al., 2004), also flown on the ER-2 during TC4.

A priori profile information is obtained from CloudSat (Stephens et al., 2008) project files of radar reflectivity, CALIPSO lidar cloud fraction, and ECMWF profiles of temperature and relative humidity. These profiles are combined with cloud microphysical probability distributions derived from in situ cloud measurements that describe ice cloud parameters, relative humidity, and liquid cloud parameters in ice clouds. The a priori information is transformed to cumulative distribution functions (CDFs) and empirical orthogonal functions (EOFs) for temperature, relative humidity, and five hydrometeor parameters at specified layers in the atmosphere. The CDFs capture the complete single-point statistics of the seven parameters, while the EOFs (from a type of rank correlation matrix) capture some of the relationships in the two-point statistics between different parameters and layers.

This Bayesian algorithm uses a hybrid Monte Carlo integration and optimization approach to retrieve quantities with uncertainty estimates. The MCI method is highly efficient because the retrieval database is precomputed, and so the radiative transfer does not have to be computed for each new observation. MCI does not require the prior pdf to have a particular functional form, such as the Gaussian distributions assumed in optimal estimation. The biggest problem with $\mathrm{MCI}$ is that, for a finite size retrieval database, increasing the length of the observation vector or making the observation uncertainties smaller results in fewer database cases 
with a significant contribution to the Monte Carlo integrals. The hybrid approach developed here uses MCI, but, if not enough database cases match the observation, an optimization is performed to maximize the posterior pdf. While much slower than MCI, the optimization minimizes a least squares cost function (assuming a Gaussian likelihood function) using gradient information to be most efficient. There is also an option to generate the Bayesian posterior distribution using the Markov chain Monte Carlo (MCMC) method, though this is only practical for testing purposes on a small number of observations. The optimization method and the generation of a large number of retrieval database cases (e.g., $10^{6}$ ) for MCI requires using an explicit prior probability distribution rather than using CloudSat profiles individually. Another advantage of using an explicit prior probability distribution is that there is some ability to extrapolate beyond the particular radar profile input.

Ice particle size distributions are defined using the particle mass as expressed by the equivalent mass sphere diameter, $D_{\mathrm{e}}$. The characteristic particle size is the IWC weighted mean $D_{\mathrm{e}}$, and the width of the size distribution is measured by the $D_{\mathrm{e}}$ dispersion:

$D_{\text {me }}=\frac{\int N\left(D_{\mathrm{e}}\right) D_{\mathrm{e}}^{3} D_{\mathrm{e}} \mathrm{d} D_{\mathrm{e}}}{\int N\left(D_{\mathrm{e}}\right) D_{\mathrm{e}}^{3} \mathrm{~d} D_{\mathrm{e}}}$

$D_{\mathrm{e}} \operatorname{disp}=\frac{1}{D_{\mathrm{me}}}\left[\frac{\int N\left(D_{\mathrm{e}}\right) D_{\mathrm{e}}^{3}\left(D_{\mathrm{e}}-D_{\mathrm{me}}\right)^{2} \mathrm{~d} D_{\mathrm{e}}}{\int N\left(D_{\mathrm{e}}\right) D_{\mathrm{e}}^{3} \mathrm{~d} D_{\mathrm{e}}}\right]^{1 / 2}$.

Hydrometeor layers above the freezing level can contain ice particles, specified by IWC, $D_{\mathrm{me}}$, and $D_{\mathrm{e}}$ dispersion, and liquid cloud droplets, specified by liquid water content (LWC), $D_{\text {me }}$, and a fixed $D_{\mathrm{e}}$ dispersion. The ice particles are a mixture of different shape categories, with the mixing fractions varying in the retrieval database. Below the freezing level, a simple thermodynamical melting model (with no vertical air motions) is used to calculate the melt fraction of the particles (see Appendix B4). The a priori microphysics for the melting/melted particles is that of the ice particles at $273 \mathrm{~K}$. The profiles of IWC, $D_{\mathrm{me}}$, and $D_{\mathrm{e}}$ dispersion describe the ice particles above the freezing level and the melting/melted particles below.

As the new ice cloud profile retrieval algorithm is described in more detail in the sections below, it will be useful to refer to the algorithm flowchart in Fig. 1. The profile retrieval system is divided into two separate Fortran 90 programs. The first (described in Sect. 3) generates most of the a priori information and outputs a file of cumulative distribution functions and combined EOFs for profiles of temperature, relative humidity, ice and melting particle IWC, $D_{\text {me }}$, $D_{\mathrm{e}}$ dispersion, and liquid cloud LWC and $D_{\mathrm{me}}$. The second program (described in Sect. 4) uses the CDF/EOF file information to create atmosphere and hydrometeor profiles with the desired a priori pdf, simulates the observations with radiative transfer, does Monte Carlo integration Bayesian retrievals, and, when those fail, performs optimization with gradient information to maximize the posterior pdf. Preparation of the microphysical pdfs from TC4 in situ data is described in Appendix A, and generation of the scattering tables for hexagonal plate aggregates, sphere aggregates, and snowflake aggregates is discussed in Appendix B.

\section{Generation of the a priori CDF/EOF file}

The CDF/EOF generation program uses data from several sources to create the a priori information for the ice cloud retrieval system. The primary data source is CloudSat reflectivity profiles, CALIPSO lidar cloud fraction for the CloudSat range bins, and the corresponding ECMWF profiles of temperature and relative humidity. The secondary sources of a priori information are parameters of several probability distributions obtained from in situ aircraft probes that describe relationships between ice cloud parameters, liquid cloud parameters, and relative humidity. Multiple profiles of IWC/LWC, $D_{\mathrm{me}}$, and $D_{\mathrm{e}}$ dispersion for ice and liquid hydrometeors are generated for each CloudSat radar profile. Radar reflectivity does not uniquely specify ice cloud microphysical parameters, of course, so radar reflectivity is combined with the ice particle microphysical statistics.

\subsection{Inputs}

Any number of CloudSat granules (GEOPROF, GEOPROFLIDAR, and ECMWF-AUX files of one orbit each) may be input, and those profiles in a selected latitude-longitude box are used. All columns in the designated region or only ones deemed cloudy may be used. The altitudes of the layer interfaces (also called levels) for analysis and output to the CDF/EOF file are specified. The ECMWF temperature and relative humidity profiles are interpolated to the specified levels. Hydrometeors are allowed in a specified subset of the layers, and the radar reflectivity and lidar cloud fraction are averaged/interpolated to each layer. CloudSat reflectivity within three range gates of the surface elevation is not used.

Parameters of three microphysical probability distributions are input. The most important one is a Gaussian distribution of $T, \ln ($ IWC $), \ln \left(D_{\mathrm{me}}\right)$, and $D_{\mathrm{e}}$ dispersion for ice particles (where $T$ is temperature). Parameters are input for a Gaussian distribution of $T, \ln ($ IWC), $\ln (\mathrm{LWC}), \ln \left(D_{\mathrm{me}, \mathrm{liq}}\right)$ for supercooled cloud droplets (where LWC is the liquid water content of the droplets, and $D_{\text {me,liq }}$ is the $D_{\text {me }}$ of the liquid cloud droplets). Finally, coefficients are input for the mean and standard deviation of a beta distribution of relative humidity in the presence of ice particles $(T<273 \mathrm{~K})$. These coefficients are defined by $\mathrm{RH}_{\text {mean }}=a+b T+c T^{2}+d \ln$ (IWC) and $\mathrm{RH}_{\text {stddev }}=e+f \ln ($ IWC). Appendix $\mathrm{A}$ describes the analysis of in situ cloud probes from TC4 to generate the a priori information input to the $\mathrm{CDF} / \mathrm{EOF}$ generation program. 

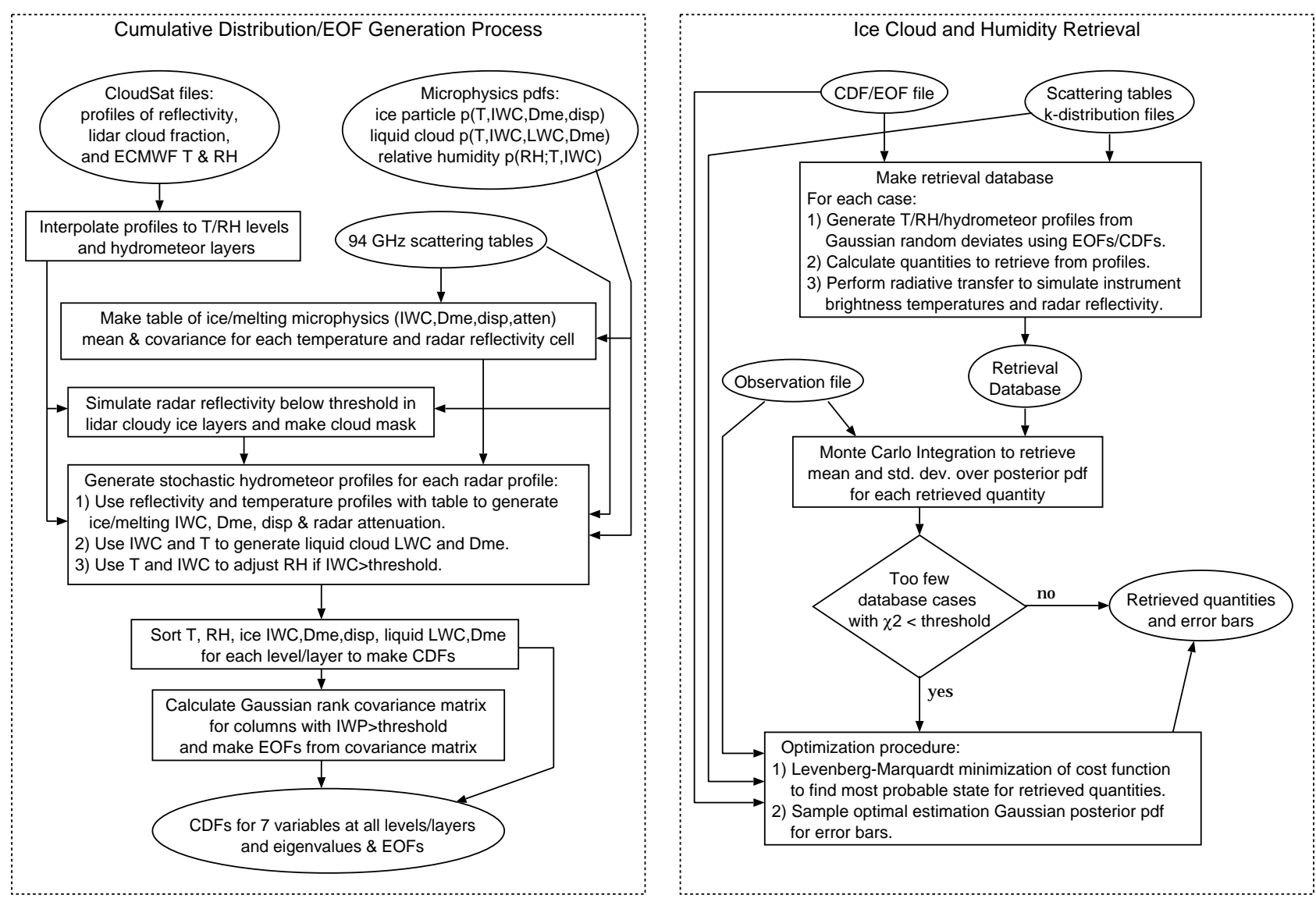

Fig. 1. Flowchart of the Bayesian ice cloud profile retrieval algorithm. Abbreviations used: " $T$ " for temperature, "RH" for relative humidity, "IWC" for ice water content, "IWP" for ice water path, "LWC" for cloud liquid water content, "Dme" for mean IWC weighted equivalent sphere diameter, "disp" for $D_{\mathrm{e}}$ dispersion (a measure of the size distribution width), "atten" for radar attenuation, "CDF" for cumulative distribution function, "EOF" for empirical orthogonal function, and "pdf" for probability density function.

Tables that specify the complete scattering information for randomly oriented particles at the $94 \mathrm{GHz}$ CloudSat radar frequency are used to relate the microphysical parameters to radar reflectivity. These tables specify the scattering properties as a function of $D_{\mathrm{me}}, D_{\mathrm{e}}$ dispersion, temperature, and particle shape. There are scattering tables for the ice particles, the melting/melted ice particles, and cloud liquid droplets. See Appendix B for a description of the particle shapes used and how these scattering tables are generated.

\subsection{Generation of the ice microphysics table}

The radar reflectivity and ice/melting particle microphysical statistics are combined by generating a two-dimensional lookup table in reflectivity and temperature (e.g., increments of $0.5 \mathrm{dBZ}$ and $2.0 \mathrm{~K}$, except $0.4 \mathrm{~K}$ in the melting zone). For each reflectivity/temperature cell of the table, the mean vector and covariance matrix of $\ln$ (IWC), $\ln \left(D_{\mathrm{me}}\right), D_{\mathrm{e}}$ dispersion, and $\ln (A)$ (where $A$ is the ice/melting particle radar attenuation coefficient in $\mathrm{dB} \mathrm{km}^{-1}$ ) are calculated. This table is made with Monte Carlo sampling of the Gaussian distribu- tion of $T, \ln (\mathrm{IWC}), \ln \left(D_{\mathrm{me}}\right), D_{\mathrm{e}}$ dispersion, random ice particle shape mixing fractions, and the appropriate scattering table (depending on $T<273 \mathrm{~K}$ or $T>273 \mathrm{~K}$ ). The eigenvalues and eigenvectors are calculated for the $4 \times 4$ covariance matrix in each reflectivity/temperature cell to be used later with the mean vector to stochastically simulate IWC, $D_{\text {me }}$, $D_{\mathrm{e}}$ dispersion, and radar attenuation consistent with the reflectivity, temperature, and the ice microphysical pdf.

\subsection{Simulation of radar reflectivity below threshold}

Visual inspection shows that a CloudSat reflectivity threshold of $-26 \mathrm{dBZ}$ for $500-\mathrm{m}$-thick layers is required to nearly eliminate spurious cloud detections due to receiver noise. This threshold needs to be higher than the nominal CloudSat sensitivity of $-30 \mathrm{dBZ}$, because the probability distribution of the receiver noise power has considerable width. Substantial amounts of ice cloud in the tropics have radar reflectivity below $-26 \mathrm{dBZ}$. A procedure is described in Appendix $\mathrm{C}$ to simulate radar reflectivity for hydrometeor layers that are below the radar threshold, but are known to be cloudy from the 

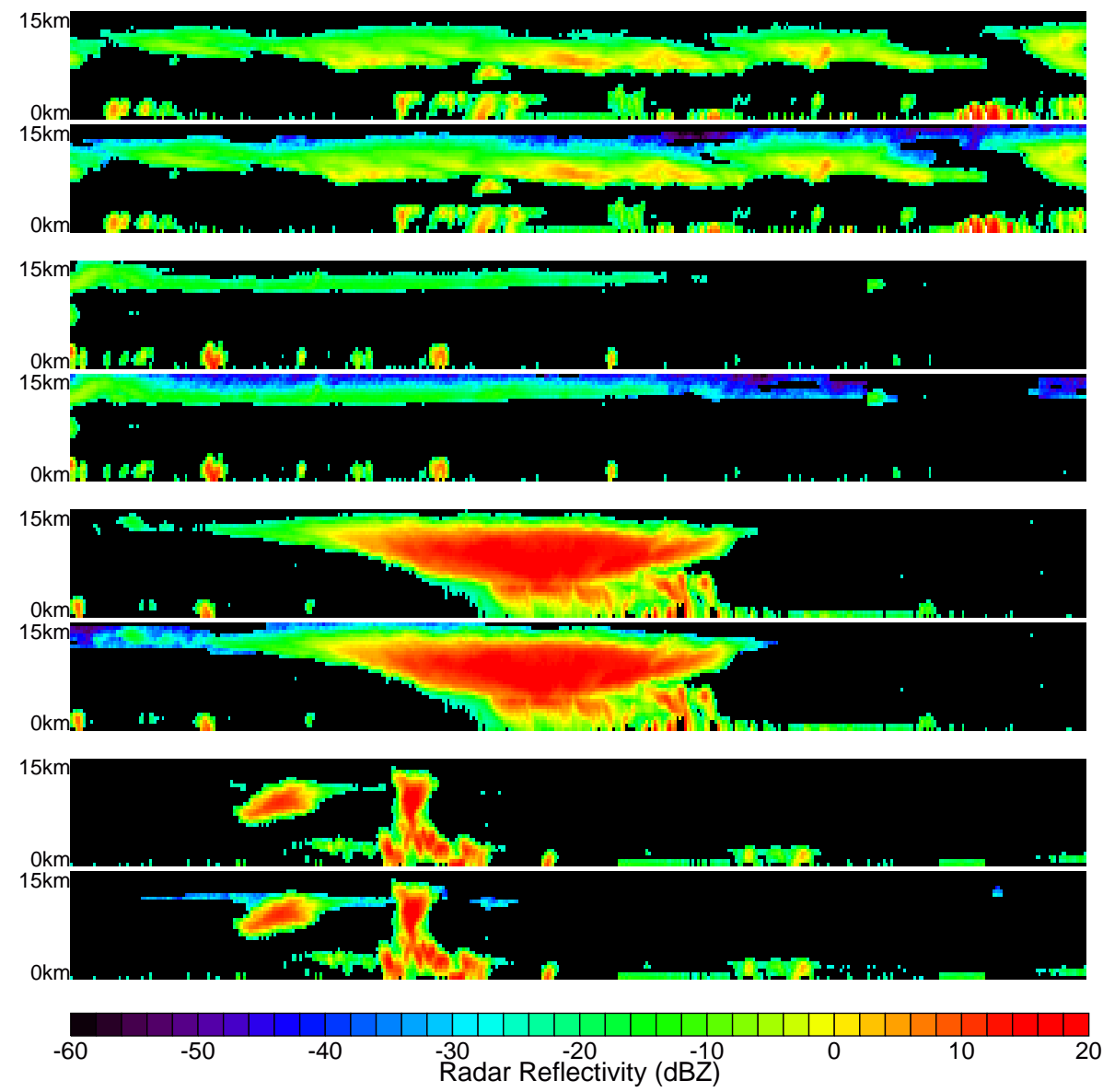

Fig. 2. An example of the stochastic simulation of radar reflectivity for lidar identified cloudy layers with CloudSat reflectivity below $-26 \mathrm{dBZ}$. For each pair of radar profiles, the top strip uses a $-26 \mathrm{dBZ}$ threshold without simulated reflectivity and the bottom strip includes the simulated reflectivity. There are 1600 CloudSat columns from two separate orbits over the TC4 region. The layers in this example are all $0.5 \mathrm{~km}$ thick.

CALIPSO lidar cloud mask. This allows the prior pdf to have lower values of IWC and $D_{\text {me }}$ than would be produced from the CloudSat reflectivity alone. An example of the radar reflectivity profiles with and without the simulated reflectivity is shown in Fig. 2.

To prevent discontinuity in the CDFs, layers identified as clear are set to very small, random reflectivity values that are correlated in the vertical. The correlation matrix for generating these fictitious reflectivity values is calculated from the radar reflectivities above the noise threshold. The mean and standard deviation of these stochastic reflectivities for clear layers are $-80 \mathrm{dBZ}$ and $1 \mathrm{dBZ}$, respectively.

\subsection{Generation of stochastic hydrometeor profiles}

The lookup table is used to generate several stochastic hydrometeor parameter profiles for each CloudSat reflectivity profile. First, the CloudSat reflectivity profiles are corrected for molecular attenuation with the absorption profile provided in the GEOPROF file (Marchand et al., 2008). For each hydrometeor profile, four independent stochastic profiles of zero mean/unit variance Gaussian deviates are generated having the same vertical correlations as the radar reflectivity. These Gaussian profiles are used with the reflectivity/temperature lookup table to stochastically generate IWC, $D_{\text {me }}, D_{\mathrm{e}}$ dispersion, and attenuation $\left(\mathrm{dB} \mathrm{km}^{-1}\right)$ that agree with the CloudSat reflectivity profile, have statistics consistent with the a priori ice microphysics distribution, and are vertically correlated like the radar profile. If a particular reflectivity is smaller than available in the lookup table, then a Rayleigh scattering extrapolation is performed. The microphysical generation process proceeds from top down, so that the generated radar attenuation (which is consistent with the hydrometeor parameters) can be applied to correct the radar reflectivity profile. If the attenuation correction exceeds $30 \mathrm{~dB}$, then the reflectivity is held constant for the rest of the profile. If the reflectivity below the melting level falls below $-20 \mathrm{dBZ}$, then the rest of the profile is set to stochastic clear sky values. Thus, the radar-based hydrometeor generation process is only used for ice particles and melting/melted 
ice particles (i.e., rain) and not for cloud droplets, boundary layer clouds or shallow convection. In deep convection, multiple scattering increases CloudSat reflectivity above the single scattering values assumed here. Battaglia et al. (2011) estimated that, in tropical deep convective cores, multiple scattering becomes important ( $>3 \mathrm{dBZ}$ ) below about $9 \mathrm{~km}$ altitude. Since correcting for multiple scattering accurately is very difficult, we simply note that the effect is to overestimate the (single scattering) $94 \mathrm{GHz}$ reflectivity when the reflectivity is high ( $>10 \mathrm{dBZ}$ ), thereby widening the a priori IWC and $D_{\text {me }}$ distributions.

Liquid cloud LWC and $D_{\text {me }}$ for layers above the melting level are generated stochastically using the Gaussian distribution in $T, \ln ($ IWC $), \ln ($ LWC $), \ln \left(D_{\text {me,liq }}\right)$ with vertical correlations according to the radar correlation matrix, but with some thresholds applied. There is no supercooled liquid cloud colder than a threshold temperature (e.g., $240 \mathrm{~K}$ to be consistent with the data used to generate the distribution). If the generated liquid cloud LWC is below $0.01 \mathrm{~g} \mathrm{~m}^{-3}$, then it is set to zero, since the Bergeron-Findeisen process would tend to eliminate small LWC in the presence of ice crystals. The cloud liquid water content and $D_{\text {me }}$ below the melting level are linearly interpolated between that of the lowest supercooled layer and the approximate lifting condensation level.

The relative humidity profile from the CloudSat ECMWF file is adjusted in the presence of significant ice water content using the coefficients of the beta distribution mean and standard deviation. The IWC has to be above a threshold (now $0.001 \mathrm{~g} \mathrm{~m}^{-3}$ ) before the relative humidity is adjusted. Instead of choosing a beta deviate randomly, the "probability" (0 to 1$)$ of the ECMWF relative humidity in its cumulative distribution is translated to the beta deviate. Thus, a low ECMWF humidity will result in a relative humidity from the low end of the beta distribution that depends on temperature and IWC. This procedure also results in the relative humidity having reasonable vertical correlations. If there is nonzero cloud liquid water content in a layer, then the relative humidity is set to $100 \%$.

\subsection{Calculating CDFs and EOFs}

Cumulative distribution functions, $D_{i}\left(x_{i}\right)$, are made by sorting (over all the stochastic hydrometeor profiles generated from the radar profiles) the temperature and relative humidity for each profile level and the hydrometeor parameters (IWC, $D_{\text {me }}, D_{\mathrm{e}}$ dispersion and liquid cloud LWC and $D_{\mathrm{me}}$ ) for each hydrometeor layer. At this point, the seven parameters for each level/layer in the profiles (generically denoted by $x_{i}$ ) are represented by the probability, $p_{i}$, or rank in the CDFs, i.e., $p_{i}=D_{i}\left(x_{i}\right)$, where $i$ specifies both the parameter and the profile level/layer.

A type of rank correlation matrix is used to generate the EOFs with the desired correlations. Thus, the joint probability distribution between two variables (e.g., IWC and
$D_{\text {me }}$ in two layers) is represented by a single number, i.e., the correlation. To make the correlations more representative of the important relationships for ice cloud retrievals, only those profiles with IWP above a specified threshold (e.g., $10 \mathrm{~g} \mathrm{~m}^{-2}$ ) are used for the correlations. This is done by having a second set of CDFs, $D_{i, \text { IWP }>}\left(x_{i}\right)$, made from those profiles with IWP above the threshold. The ranks or probabilities representing the profiles are converted to Gaussian deviates using

$\xi_{i}=\Phi^{-1}\left[D_{i, \mathrm{IWP}>}\left(x_{i}\right)\right]$,

where $\Phi(\xi)$ is the cumulative distribution function of the standard normal distribution. Gaussian distributions work best with EOFs, because linear combinations of independent Gaussian deviates remain Gaussian. The covariance matrix for the EOFs is calculated from these Gaussian deviates. Since the Gaussian deviates $\xi_{i}$ have zero mean and unit variance, the covariance matrix is also the correlation matrix:

$C_{i j}=\frac{1}{N_{\text {prof }}} \sum_{k=1}^{N_{\text {prof }}} \xi_{i}^{(k)} \xi_{j}^{(k)}$,

where the sum is over the $N_{\text {prof }}$ stochastic profiles with IWP above the threshold for the correlation matrix. The eigenvectors of the correlation matrix, $C_{i j}$, are the EOFs.

The output CDF/EOF file contains the heights of the profile levels and hydrometeor layers; the CDFs of temperature, relative humidity, IWC, $D_{\mathrm{me}}, D_{\mathrm{e}}$ dispersion, and liquid cloud LWC and $D_{\text {me }}$ for each level or layer; and the Gaussian EOF eigenvalues and eigenvectors.

\subsection{Example CDFs and rank correlation matrix}

A CDF/EOF file is produced from the 41 CloudSat orbits that have lidar cloud fraction and intersect the target region of $4^{\circ} \mathrm{N}$ to $12^{\circ} \mathrm{N}$ and $90^{\circ} \mathrm{W}$ to $80^{\circ} \mathrm{W}$ (the TC4 Pacific Ocean region) in July and August 2007. A total of 32403 radar columns (cloudy and clear) are used, with three stochastic hydrometeor profiles generated for each radar column. Of the 97209 stochastic hydrometeor profiles, 46191 have ice water path above $10 \mathrm{~g} \mathrm{~m}^{-2}$ and are used in the EOF correlation matrix. The layer thickness in most of the ice region is $0.5 \mathrm{~km}$, with $0.2 \mathrm{~km}$ resolution in the main melting region. The cumulative distribution functions are output at 201 points, and the 43 temperature/humidity levels and 32 hydrometeor layers result in an EOF vector length of 246.

Figures 3 to 5 show examples of the CDFs as profiles of each parameter for seven percentiles $(0,5,25,50,75,95$, and 100) in the cumulative distributions. The dots on the median profile show the temperature/humidity levels or the hydrometeor layer centers. The temperature profiles show the small range of temperature typical of the tropical troposphere. The relative humidities have a large possible range in the ice cloud region, though $50 \%$ are in a much smaller range that generally decreases with height in the ice cloud 
region (about 5 to $15 \mathrm{~km}$ ). The a priori IWC profiles have a tremendous range from effectively clear $\left(<10^{-5} \mathrm{~g} \mathrm{~m}^{-3}\right)$ to about $10 \mathrm{~g} \mathrm{~m}^{-3}$. Similarly, $D_{\text {me }}$ ranges from below $15 \mu \mathrm{m}$ to above $1500 \mu \mathrm{m}$, though the maximum $D_{\text {me }}$ generally decreases with height. The 95 th percentile of IWC shows that the highest IWCs are deep, ranging from the surface to about $14 \mathrm{~km}$. The highest layer cloud fraction (up to $45 \%$ for $0.5 \mathrm{~km}$ layers) is in the anvil region from 10 to $14 \mathrm{~km}$. Including the correlations between layers, the resulting cloud fraction is about $89 \%$. The peak layer liquid cloud fraction is about $11 \%$ near the melting level.

Figure 6 shows the correlation matrix used to make the EOFs with each part of the matrix labeled by the parameters. Nearby layers of temperature, relative humidity, IWC, and $D_{\text {me }}$ are highly correlated. IWC and $D_{\text {me }}$ of the same layer have a reasonably high rank correlation. The fairly high correlation between relative humidity and IWC can be seen. Although there seems to be a lot of information in the correlation matrix, and hence the EOFs, it should be noted that there is only one number to represent the relationship between any two variables, which is a tiny fraction of the information contained in a joint probability distribution. It is, however, the same amount of information contained in the multi-variate Gaussian distribution assumed in the optimal estimation method.

\section{Ice cloud/humidity profile retrieval process}

The inputs to the retrieval program are (1) the CDF/EOF file of a priori information, (2) a file of observation vectors (with measurement uncertainties and viewing directions), (3) a retrieval database file, and (4) many parameters and files that define the characteristics of the retrievals and measurements and provide data for the radiative transfer calculations. The retrieval program is run in one of two modes: (1) generate and output a retrieval database, or (2) read a retrieval database and perform retrievals. For each database "case", an atmosphere/cloud profile is generated with the desired a priori information from the CDF/EOF file information, and then the radiative transfer is done to simulate the observations.

\subsection{Atmosphere profile generation}

The atmosphere/cloud profile generation process begins with generating a vector of independent, standard Gaussian random deviates $(\xi)$, most of which are used to drive the EOFs. The number of EOFs used is user specified, and may be chosen, for example, to include $99 \%$ of the variance. The Gaussian deviates are multiplied by the square root of the eigenvalues to make the random EOF coefficients, which are then multiplied by the eigenvector matrix to give a long vector of correlated Gaussian distributed elements. Gaussian random deviates are used because the Gaussian distribution shape is preserved upon the linear EOF transforma-
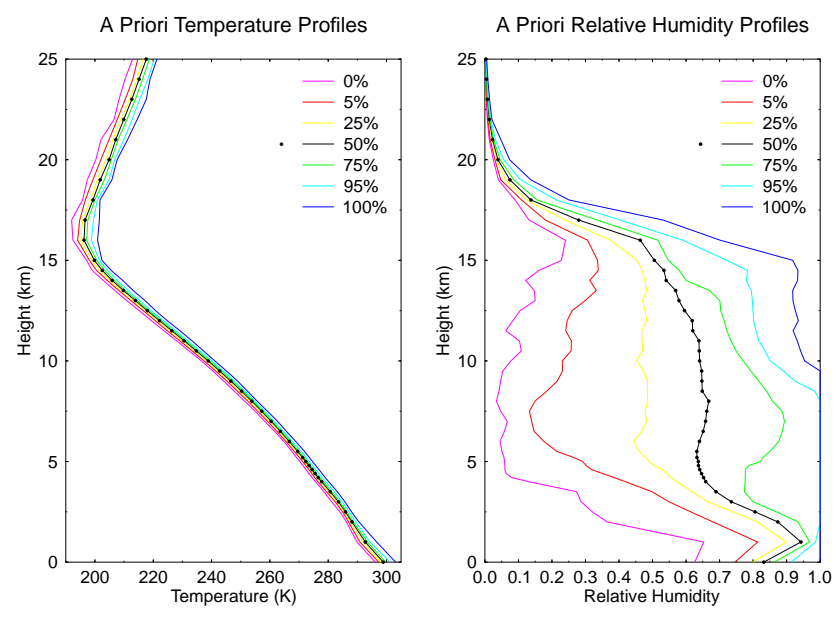

Fig. 3. Profiles of temperature and relative humidity from seven percentiles in the a priori cumulative distribution functions.

tion. These Gaussian distributed elements are converted to probabilities, $p_{i}$, with uniform distributions between 0 and 1 using the standard normal cumulative distribution function, i.e., $p_{i}=\Phi\left(\xi_{i}\right)$. These correlated "probabilities" are then used to index into the temperature, relative humidity, and hydrometeor CDFs to produce the correlated profiles, i.e., $x_{i}=D_{i}^{-1}\left(p_{i}\right)$.

The relative humidities are converted to water vapor mixing ratio $q$, since that is what the radiative transfer routines require. This conversion requires the pressure profile, which is derived from the temperature profile using the hypsometric equation. If the relative humidity profile is to be a retrieved quantity, then desired levels are stored in the retrieval vector.

The hydrometeor profiles are IWC, $D_{\text {me }}$, and $D_{\mathrm{e}}$ dispersion for the ice/melted particles, and the LWC and $D_{\text {me }}$ for cloud liquid droplets. If the layer temperature is below the melting temperature, the IWC and $D_{\text {me }}$ are used for the ice particle component, and, if the temperature is above the melting temperature, the IWC and $D_{\text {me }}$ are used for the melting/melted particle component. To preserve differentiability, this temperature threshold is implemented with a smooth exponential that quickly varies between 0 and 1 . The liquid cloud droplet LWC and $D_{\mathrm{me}}$ are applied to the third component, and the $D_{\mathrm{e}}$ dispersion is set to 0.3 . In the radiative transfer, each hydrometeor component (ice, melting, liquid droplets) is associated with its own set of scattering tables (one for each frequency).

Another set of $\left(N_{\text {layer,shape }}+1\right) N_{\text {shape }}$ Gaussian deviates (from $\xi$ ) is used to control the ice particle shape mixing fraction of the $N_{\text {shape }}$ possible shapes in the scattering tables at $N_{\text {layer,shape }}+1$ heights in the hydrometeor profiles. Testing finds that linear interpolation over the whole ice region $\left(N_{\text {layer,shape }}=1\right)$ gives an adequate number of degrees of freedom for particle shape. The Gaussian deviates are converted to uniformly distributed deviates and linearly interpolated in 

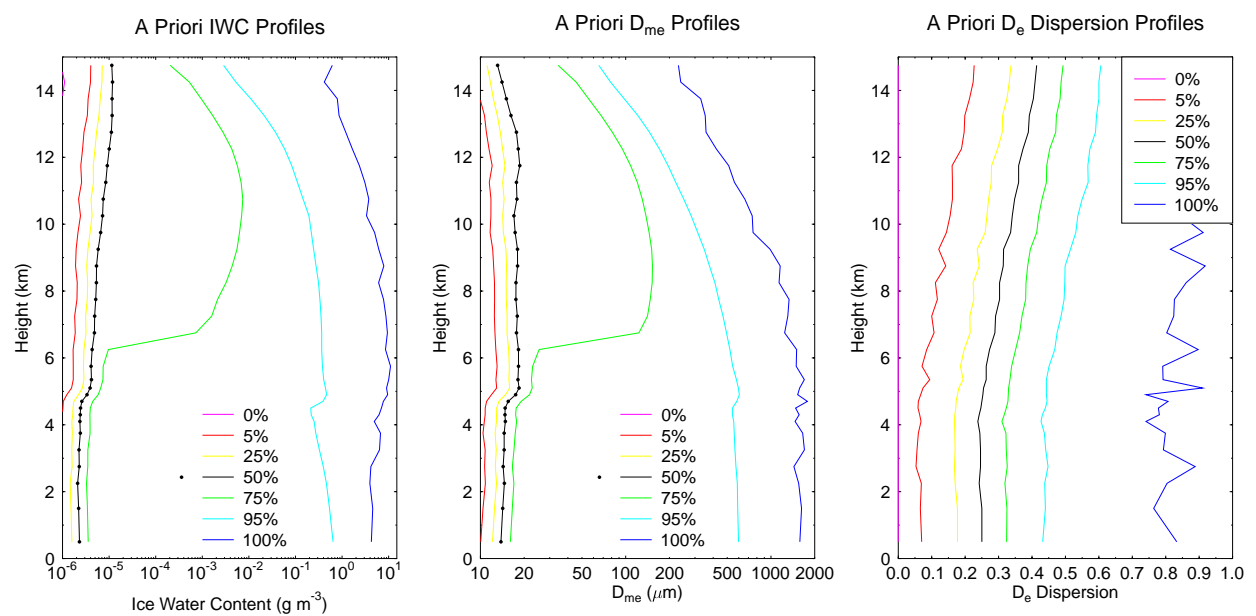

Fig. 4. Profiles of IWC, $D_{\mathrm{me}}$, and $D_{\mathrm{e}}$ dispersion from seven percentiles in the a priori cumulative distribution functions. Below the freezing level, the profiles are applied to the melting/melted particles instead of the ice particles.
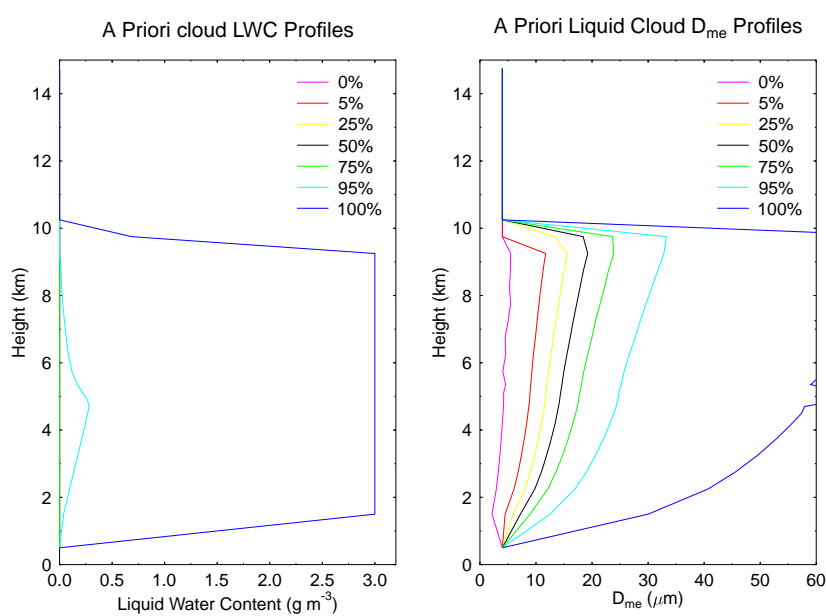

Fig. 5. Profiles of liquid cloud LWC and $D_{\text {me }}$ from seven percentiles in the a priori cumulative distribution functions.

height to $N_{\text {shape }}$ fractions at each hydrometeor layer. These fractions are then adjusted according to the layer $D_{\text {me }}$ so that the mixing fraction is set to zero for particle shapes for which the $D_{\mathrm{me}}$ is outside the range in the scattering tables (and the shape mixing fractions sum to 1). Thus, the particle shape mixing fractions are not controlled by the a priori CDF/EOF process, but are considered completely unknown prior to the observations. There is, however, prior information about the particle shape in that only certain shapes are available at a given $D_{\mathrm{me}}$; for example, hail is only available for $D_{\mathrm{me}}>398 \mu \mathrm{m}$.

Four vertically integrated quantities, IWP, average $D_{\text {me }}$, median IWP height $\left(Z_{\mathrm{med}}\right)$, and melted liquid water path (LWP), are calculated from the IWC and $D_{\text {me }}$ profiles and stored in the retrieval vector. The IWC weighted shape mixing fractions and cloud droplet LWP are also stored in the

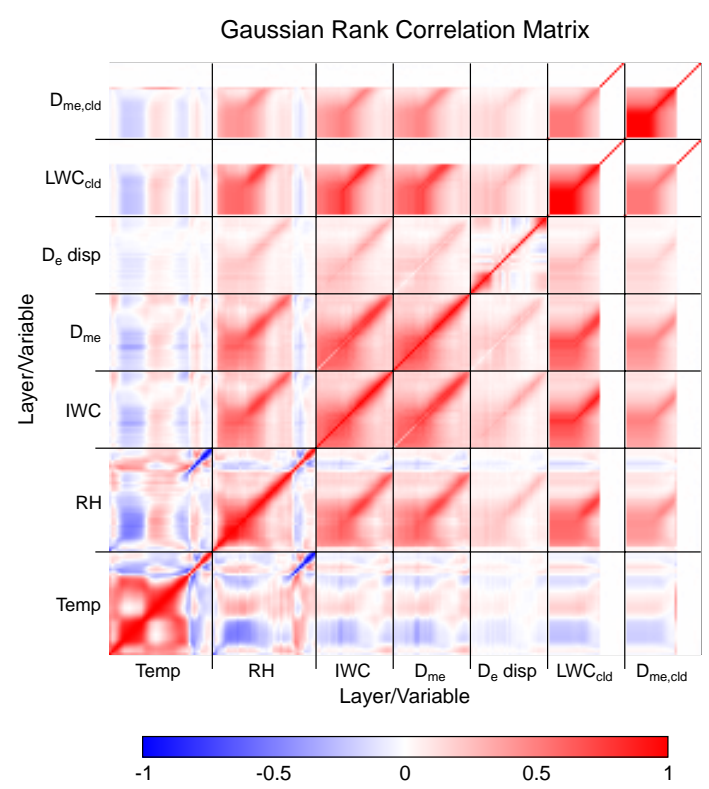

Fig. 6. The Gaussian correlation matrix used to make the EOFs. Blue is a correlation of -1 , white a correlation of 0 , and red a correlation of 1. Each element of the matrix represents a pair of parameters and layers, with increasing height of the levels/layers within each parameter block. The $T$ and RH levels from the surface up are shown as dots in Fig. 3, while the hydrometeor layer centers are shown as dots in Fig. 4.

retrieval vector. If desired, the IWC and $D_{\text {me }}$ profiles at a specified resolution are stored in the retrieval vector.

\subsection{Radiative transfer}

The second part of making the retrieval database is the radiative transfer. Currently, the 1-D radiative transfer for passive radiometers is calculated with SHDOMPPDA (Evans, 2007), 
though there could be options for other radiative transfer models in the future. SHDOMPPDA was chosen because an adjoint, which is needed for the optimization, was already developed. It can perform unpolarized solar and thermal emission radiative transfer for randomly oriented particles, and is flexible in the trade-off between accuracy and computational efficiency. The radiative transfer module also supports calculating radar reflectivity profiles and vertically integrated backscattering, which includes attenuation by gases and particles, but not multiple scattering. A k-distribution system is used to calculate molecular absorption given the profiles of temperature, pressure, water vapor and ozone mixing ratio. The script for generating the k-distribution table for each channel with LBLRTM (Clough et al., 2005) includes the spectral response of the channel. For CoSSIR and CRS a fourth-order Butterworth filter response is assumed for the bandpasses, and, of course, only one " $k$ " is needed for these nearly monochromatic channels. The surface reflection is simply specified with the mean and standard deviation of the stochastically varied Lambertian emissivity, which is either completely correlated or uncorrelated between channels. A few elements of the Gaussian deviate vector $\boldsymbol{\xi}$ are used to vary the surface emissivity.

The single scattering properties are input with a scattering table for each channel. The scattering tables have the extinction, single scattering albedo, and Legendre series of the phase function tabulated as a function of $D_{\mathrm{me}}, D_{\mathrm{e}}$ dispersion, particle shape, and temperature. The temperature dependence is needed in the microwave, especially for liquid water, but also for the weak absorption of ice. The range of $D_{\text {me }}$ tabulated varies with particle shape. The optical properties are interpolated in $\ln \left(D_{\mathrm{me}}\right), D_{\mathrm{e}}$ dispersion, and temperature using successive cubic splines. The gradients of cubic spline interpolation are continuous, which is important for the gradient-based optimization calculation. A trilinear interpolation option is also available if optimization is not going to be used. Interpolation is performed on log extinction, single scattering albedo, and the Legendre coefficients.

\subsection{MCI and optimization retrieval methods}

The primary and most efficient solution method used in the ice cloud profile Bayesian retrieval algorithm is Monte Carlo integration (MCI) (e.g., Evans et al., 2002). Random atmospheric/cloud profiles distributed according to the a priori pdf are generated as described above. The retrieval database contains the desired retrieved quantities (e.g., IWP, mean $D_{\text {me }}$, $Z_{\text {med }}$ and perhaps IWC $/ D_{\text {me }}$ and relative humidity profiles) and the associated simulated observations (e.g., brightness temperature for each channel and a radar reflectivity profile) for one or more viewing angles. Specified simulated channels in the retrieval database may be treated as retrieved parameters, so that one instrument may be used to retrieve observables of another for comparison.
The MCI algorithm calculates the conditional pdf (or likelihood function) in Bayes' theorem, which is assumed to be an uncorrelated Gaussian distribution in the difference between the simulated and measured observations. Since the database cases are distributed according to the a priori pdf, the conditional pdf is the same as the posterior pdf:

$p_{\text {post }} \propto \exp \left(-\frac{1}{2} \chi^{2}\right) \quad \chi^{2}=\sum_{j=1}^{N_{\text {chan }}} \frac{\left(y_{j}^{(\text {sim })}-y_{j}^{(\text {obs })}\right)^{2}}{\sigma_{j}^{2}}$

where $y_{j}^{(\mathrm{sim})}$ and $y_{j}^{(\mathrm{obs})}$ are the simulated and measured observations in channel $j$ with a combined measurement and forward modeling uncertainty of $\sigma_{j}$. The retrieved quantities (e.g., IWP) are the posterior pdf weighted means,

$W_{\text {ret }}=\frac{\sum_{i} W_{i} \exp \left(-\frac{1}{2} \chi_{i}^{2}\right)}{\sum_{i} \exp \left(-\frac{1}{2} \chi_{i}^{2}\right)}$,

and the retrieved uncertainty is the weighted standard deviation. There is an option to perform the integration in $\log$ space for the cloud parameters (IWC and $D_{\text {me }}$ ). The probability of an ice cloud is retrieved from the ratio of the sum of the posterior probability of cases using

$p_{\text {cloud }}=\frac{\sum_{i} \exp \left(-\frac{1}{2} \chi_{i}^{2}\right) \text { for } \mathrm{IWP}_{i}>\mathrm{IWP}_{\text {clear }}}{\sum_{i} \exp \left(-\frac{1}{2} \chi_{i}^{2}\right)}$.

If a particular measurement is flagged as bad, then the corresponding $\sigma_{j}$ is multiplied by 1000 to effectively ignore it. For a cross-track scanning instrument, there are multiple viewing angles stored in the retrieval database and the simulated observations are interpolated to the actual observation viewing angle. If the number of database cases with an $\chi^{2}$ less than a user-specified threshold is below a specified number, then the Monte Carlo integration is said to have failed, and the slower optimization process is begun for that observation vector.

The control vector for the optimization is the independent Gaussian deviates $(\xi)$, not the geophysical variables derived from them in making the atmosphere/cloud profiles. This is natural given the connection to the Monte Carlo integration retrieval method, and means that the atmosphere/cloud/surface a priori information is contained in the function that relates the geophysical variables $(\boldsymbol{x})$ to the control vector, i.e., $\boldsymbol{x}=\mathbf{G}(\boldsymbol{\xi})$. In data assimilation this approach is called control variable transformation (Bannister, 2008). If the radiative transfer function is $F_{j}(\boldsymbol{x})$, the posterior pdf is then maximized by minimizing the cost function $(J)$ :

$$
J=\sum_{i=1}^{N_{\text {dim }}} \xi_{i}^{2}+\sum_{j=1}^{N_{\text {chan }}} \frac{\left(y_{j}^{(\text {obs })}-F_{j}[\mathbf{G}(\boldsymbol{\xi})]\right)^{2}}{\sigma_{j}^{2}} .
$$


The first term is the formal background or a priori from the independent Gaussian distribution of the state vector elements $\xi_{i}$, while the second term is the observational $\chi^{2}$. This assumes that the observational uncertainties combined with the radiative transfer errors are Gaussian and independent in each channel. Internal to the retrieval program, the database stores the control vectors $(\xi)$, and the optimization is initialized with the $\xi$ of the case having the minimum $\chi^{2}$.

The advantage of having the cost function in the least squares form is that the robust and efficient LevenbergMarquardt minimization method may be used. The quadratic form of the formal background term in the cost function also means that the optimal estimation framework of Rodgers (2000) may be applied even though the real a priori distribution (contained in $\mathbf{G}(\boldsymbol{\xi})$ ) is highly non-Gaussian. The Levenberg-Marquardt formulation described in Rodgers (2000) (Sect. 5.7) is implemented. This requires the Jacobian of the forward function $\mathbf{F}[\mathbf{G}(\boldsymbol{\xi})$ ], or the $\mathbf{K}$ matrix in the notation of Rodgers. The $\mathbf{K}$ matrix is calculated using the adjoint of the radiative transfer for each channel $\left(F_{j}(\boldsymbol{x})\right)$ and the adjoint of the a priori function $\mathbf{G}(\boldsymbol{\xi})$ that calculates the geophysical variables from the control vector.

The optimization process to find the retrieval solution does not provide the uncertainties of the retrieval, as the Monte Carlo integration method does. Therefore, the local Gaussian approximation used in the optimal estimation framework (Rodgers, 2000) is implemented to distribute points around the solution $\left(\xi_{\min }\right)$ in control vector space to characterize the retrieval uncertainty. The local Gaussian approximation method computes the optimal estimation error covariance matrix. This assumes that the forward function $\mathbf{F}[\mathbf{G}(\boldsymbol{\xi})]$ is only moderately non-linear, so that the linearization and Gaussian distribution assumptions are valid. The optimal estimation posterior error covariance matrix is

$\mathbf{S}=\left(\mathbf{S}_{\mathrm{a}}^{-1}+\mathbf{K}^{T} \mathbf{S}_{y}^{-1} \mathbf{K}\right)^{-1}$

where $\mathbf{K}$ is the linearization matrix of $\mathbf{F}[\mathbf{G}(\xi)], \mathbf{S}_{y}$ the observational error covariance matrix, assumed here to be diagonal with elements $\sigma_{j}^{2}$, and $\mathbf{S}_{\mathrm{a}}$ is the a priori error covariance matrix, which here is the covariance of $\boldsymbol{\xi}$ and is thus the identity matrix. The Cholesky decomposition of $\mathbf{S}$ is used to generate random points with a correlated Gaussian distribution around $\xi_{\min }$. Of course, we are not interested in the error characterization in control vector space, so the random points in $\xi$ are transformed to the atmosphere/cloud profiles and a Monte Carlo integration is done over the retrieval quantities. Since this step does not involve radiative transfer, a large number of Monte Carlo samples (e.g., 1000) can be used in the integration.

\subsection{Markov chain Monte Carlo solution method}

The Markov chain Monte Carlo (MCMC) technique is developed as an optional solution method to check the accuracy of the optimization/local Gaussian approximation method. The MCMC has been applied in atmospheric remote sensing for several low-dimensional retrieval problems (e.g., Tamminen and Kyrola, 2001; Posselt et al., 2008). Here a more advanced "stochastic approximation adaptive" MCMC approach, basically Algorithm 4 in Andrieu and Thoms (2002), is used. The MCMC method creates a Markov chain of control vectors $\xi_{i}$ that are distributed according to the Bayesian posterior distribution, which here is $\exp (-J / 2)$. The Markov chain is started at the optimization end point $\left(\boldsymbol{\xi}_{\min }\right)$, so iterations are not wasted finding the high probability region of the control vector space. The basic Metropolis algorithm step of MCMC is to calculate a trial control vector point in the Markov chain $\left(\xi_{\text {trial }}\right)$ from the current point $\left(\xi_{i}\right)$ using a symmetric "proposal distribution" centered on $\boldsymbol{\xi}_{i}$. The cost function, $J\left(\xi_{\text {trial }}\right)$, at the trial point is computed and compared to the current point cost function, $J\left(\xi_{i}\right)$, by defining an acceptance ratio, $\alpha$ :

$\alpha=\operatorname{MIN}\left\{1, \exp \left(-\frac{1}{2}\left[J\left(\xi_{\text {trial }}\right)-J\left(\xi_{i}\right)\right]\right)\right\}$.

The trial point is accepted, $\boldsymbol{\xi}_{i+1}=\boldsymbol{\xi}_{\text {trial }}$, if a uniform random number is less than the acceptance ratio, and otherwise $\xi_{i+1}=\xi_{i}$ (the current point is reused). In adaptive MCMC the proposal distribution is updated according to the history of the Markov chain, i.e., the $\xi_{i}$ vectors. As is usually done, here the proposal distribution is taken to be a multivariate Gaussian distribution with covariance matrix $\lambda_{i} \Sigma_{i}$, so that $\xi_{\text {trial }}$ is sampled from $\mathcal{N}\left(\xi_{i}, \lambda_{i} \Sigma_{i}\right)$. The "stochastic approximation" part of the adaptive MCMC algorithm is that the mean $\left(\boldsymbol{\mu}_{i}\right)$ and covariance matrix $\left(\Sigma_{i}\right)$ of the control vectors are continuously updated according to a relaxation parameter $\gamma$ that smoothly decreases to zero:

$$
\begin{aligned}
\boldsymbol{\mu}_{i+1} & =\boldsymbol{\mu}_{i}+\gamma_{i+1}\left(\boldsymbol{\xi}_{i+1}-\boldsymbol{\mu}_{i}\right) \\
\Sigma_{i+1} & =\Sigma_{i}+\gamma_{i+1}\left[\left(\boldsymbol{\xi}_{i+1}-\boldsymbol{\mu}_{i}\right)\left(\boldsymbol{\xi}_{i+1}-\boldsymbol{\mu}_{i}\right)^{T}-\Sigma_{i}\right] .
\end{aligned}
$$

Since this updating formula is not guaranteed to keep the covariance matrix positive definite and the Cholesky decomposition of $\Sigma$ is needed to generate the random trial points, actually the Cholesky decomposition is updated. The Cholesky updating formula in Sect. 5.1.1 of Andrieu and Thoms (2002) is incorrect, so an algorithm that is first-order in $\gamma$ was derived from the Cholesky decomposition algorithm. The proposal distribution covariance scaling parameter $\lambda_{i}$ is also updated with a stochastic approximation formula:

$\lambda_{i+1}=\lambda_{i} \exp \left[\gamma_{i+1}\left(\alpha-\alpha_{*}\right)\right]$,

so that the mean acceptance ratio converges to the target $\alpha_{*}=0.234$. Here the relaxation parameter $\gamma$ is decreased according to a power law in the iteration number $i$, specified by the power and final relaxation parameter value (though $\gamma$ is not allowed to exceed 0.01). After a "burn in" fraction of the total number of iterations, the proposal distribution updating is stopped and the MCMC points are used in a Monte 

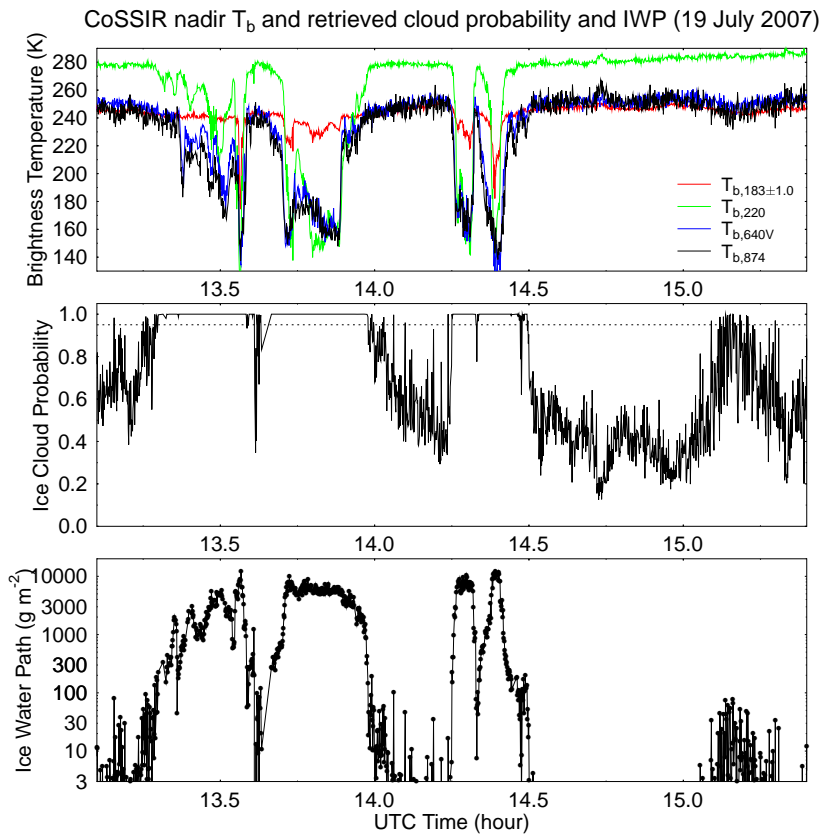

Fig. 7. CoSSIR brightness temperatures for four channels (top panel). Retrieved probability of ice cloud (middle panel). A dotted line at 0.95 probability is shown. Retrieved ice water path (bottom panel).

Carlo integration over the posterior distribution to calculate the mean parameters for the retrievals and the standard deviations for the errors. In this application the number of iterations must be very large $\left(>10^{5}\right)$ so that the MCMC solution method is impractical for use on whole datasets. The MCMC method is, however, useful for comparison with the other solution methods for a small number of pixels.

\section{Example retrieval results}

During TC4, CoSSIR measured brightness temperatures in channels around 183.3, 220.0, 380.2, 640.0 and $873.6 \mathrm{GHz}$, all with matched beamwidths of about $4^{\circ}$. The CoSSIR scanning pattern consisted of three parts during each $10 \mathrm{~s}$ scan: forward and aft conical scans and two quick crosstrack scans through nadir. The beam dwell (integration) time is $100 \mathrm{~ms}$ for the conical scans, but only $10 \mathrm{~ms}$ for nadir view, which results in about three times the receiver noise for nadir pixels. Due to hardware problems during the campaign, CoSSIR data from ice clouds are available only for the 17 July, 19 July, and 8 August flights. The Cloud Radar System (CRS) is a Doppler, polarimetric radar, though only the reflectivity profiles from the NASA Earth Science Project Office (ESPO) archive are used here. The CRS antenna beamwidth is $0.6^{\circ}$ by $0.8^{\circ}$ and views nadir. The CRS sensitivity is $-29 \mathrm{dBZe}$ at $10 \mathrm{~km}$ range with $150 \mathrm{~m}$ range resolution and $1 \mathrm{~s}$ averaging.
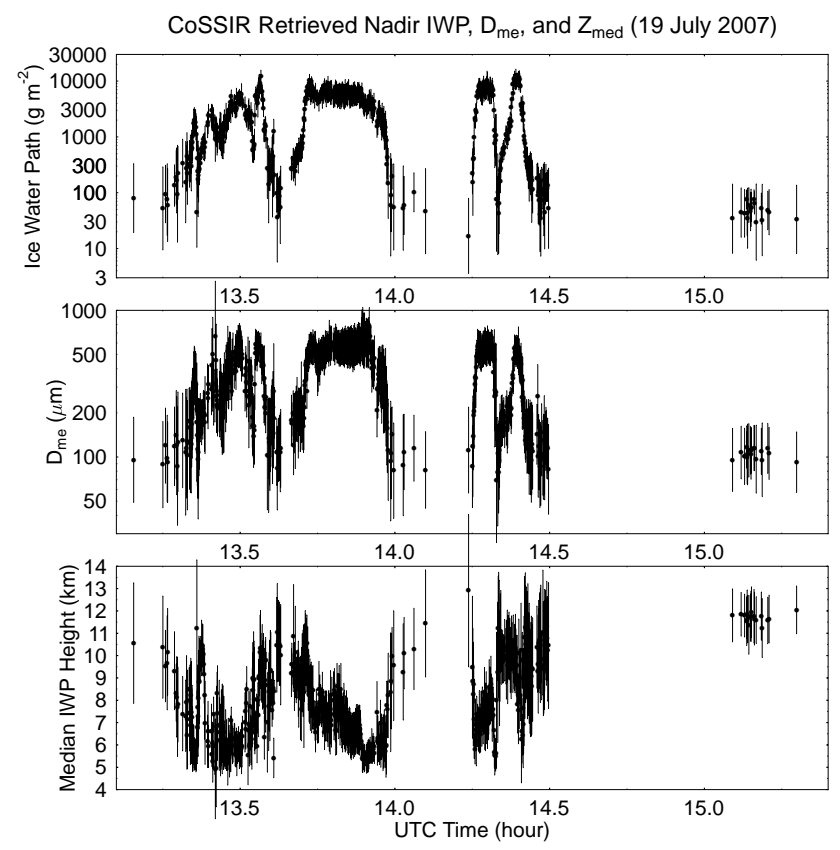

Fig. 8. CoSSIR retrieved ice water path (top panel), $D_{\text {me }}$ (middle panel), and median IWP height (bottom panel) for cloudy nadir pixels of the 19 July flight. $1-\sigma$ error bars are shown for each retrieved quantity.

Retrievals are performed mostly with CoSSIR nadir viewing data obtained 19 July from 9 CoSSIR channels $(183.3 \pm 1.0,3.0,6.6,220,380.2 \pm 1.8,3.3,6.2,640 \mathrm{~V}$, and $874 \mathrm{GHz})$. The CoSSIR uncertainties $(\sigma \mathrm{s})$, obtained from calibration target fluctuation statistics, are 1.60, 1.62, 1.59, $1.59,2.00,2.45,2.36,2.38,4.03 \mathrm{~K}$ for the 9 channels used on 19 July. Retrievals from CoSSIR brightness temperatures are also done for the same 9 channels on 17 July and for 6 channels on 8 August (without the $380 \mathrm{GHz}$ channels because the $380 \mathrm{GHz}$ receiver failed). The CoSSIR uncertainties $(\sigma \mathrm{s})$ are somewhat different for these two other flights. The CoSSIR channels are used to directly retrieve $94 \mathrm{GHz}$ radar reflectivity profiles. The profile retrieval algorithm is also used to operate on CRS reflectivity profiles alone or with CoSSIR nadir data. When CRS radar reflectivity is input to the retrieval, it is averaged from $75 \mathrm{~m}$ resolution to $500 \mathrm{~m}$ (20 layers from 5 to $15 \mathrm{~km}$ ) and has a multiplicative uncertainty of 0.4 (an estimated calibration uncertainty of about $1.5 \mathrm{~dB}$ ). The CRS radar additive uncertainty is calculated from a clear region, and ranges from about $0.0028 \mathrm{~mm}^{6} \mathrm{~m}^{-3}(-25.5 \mathrm{dBZ})$ near the surface to about $0.0003 \mathrm{~mm}^{6} \mathrm{~m}^{-3}(-35 \mathrm{dBZ})$ at $15 \mathrm{~km}$.

The retrievals are performed using the CDF/EOF file, for which results are shown in Sect. 3. The first 146 EOFs of the total of 246 , which have $99 \%$ of the variance, are used. Monte Carlo integration retrievals are done with a retrieval database of $10^{6}$ cases. At least 25 cases with a reduced $\chi^{2}$ less than 2 are required for a successful integration retrieval (and no more than 1000 are used to reduce computation). The 


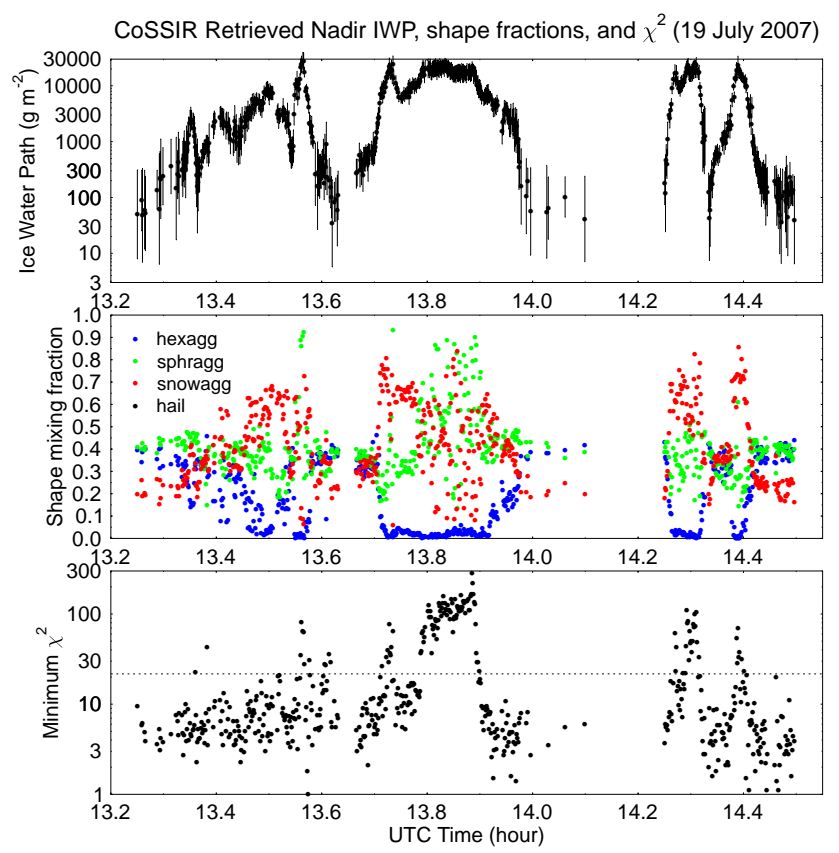

Fig. 9. Nadir retrievals of ice water path with error bars (top panel), shape mixing fractions (middle panel), and minimum $\chi^{2}$ (bottom panel) for the retrieval with three particle shapes. The dotted line at $\chi^{2}$ of 21.7 is the $99 \%$ significance level for 9 channels.

SHDOMPPDA radiative transfer is done with four discrete ordinates in zenith angle. The mean ocean surface emissivity is that of flat water (from the Fresnel formula) plus 0.06 to approximate ocean roughening, and the standard deviation of surface emissivity is 0.03 . The ice scattering tables usually have four particle shapes (hexagonal plate aggregates, sphere aggregates or graupel, dendrite aggregates, and solid spherical hail), but some results are shown for retrievals with three shapes (excluding the hail). See Appendix B for the rationale and details about these particle shapes. Scattering tables are also input for the melting/melted particles corresponding to the ice particles, and for liquid cloud droplets. The relative humidity profile from 1 to $15 \mathrm{~km}$ and 21 layer profiles of IWC and $D_{\text {me }}$ are retrieved (from 4.5 to $15 \mathrm{~km}$ ). The cloud parameter retrievals are done in log-space, which means that the uncertainties are standard deviations of $\ln$ (IWC) and $\ln \left(D_{\mathrm{me}}\right)$ (translated into 1-sigma error ranges for the plots).

Except as noted, all retrievals below use the hybrid approach of Monte Carlo integration followed by cost function optimization if too few database cases match the observations. Figure 7 shows selected CoSSIR nadir brightness temperatures, the retrieved probability of cloud, and the ice water path. The correspondence between the brightness temperature depressions and the retrieved IWP is easily seen. The retrieved probability of ice cloud is the Bayesian cloud probability given the CoSSIR measurements, because the a priori data include all CloudSat profiles (clear and cloudy). Somewhat arbitrarily, a retrieved probability thresh-

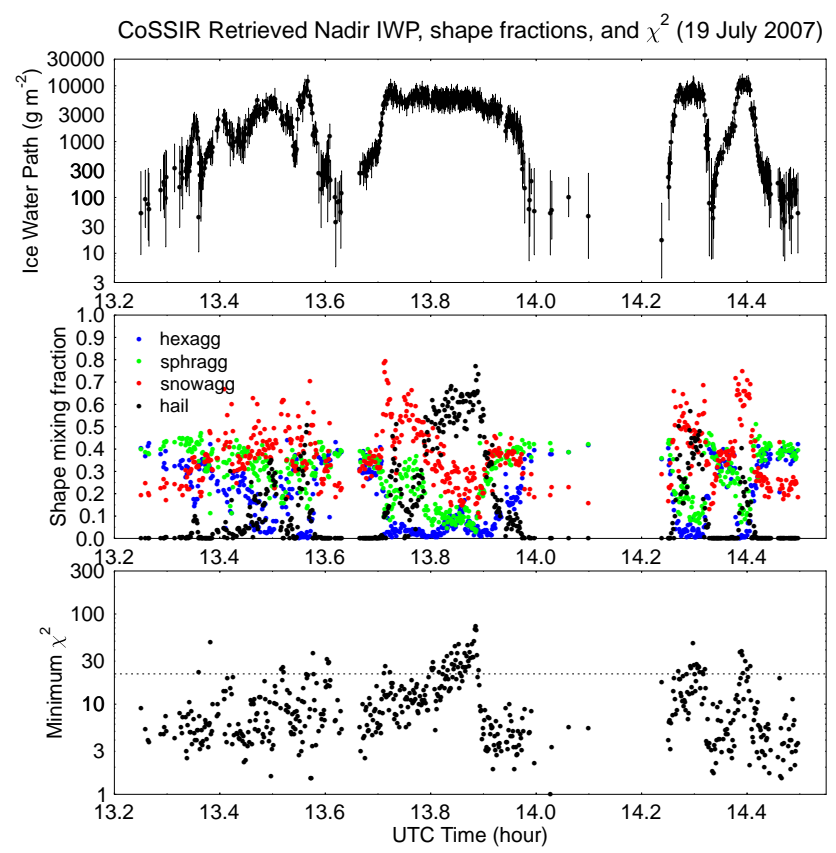

Fig. 10. Nadir retrievals of ice water path with error bars (top panel), shape mixing fractions (middle panel), and minimum $\chi^{2}$ (bottom panel) for the retrieval with four particle shapes.

old of 0.95 is chosen to indicate cloud. The retrieved ice water path, $D_{\text {me }}$, and median IWP cloud height, all with error bars, for the cloudy pixels on 19 July are shown in Fig. 8. Over the 568 cloudy pixels, the IWP ranges from $35 \mathrm{~g} \mathrm{~m}^{-2}$ to about $11000 \mathrm{~g} \mathrm{~m}^{-2}$ (1 to 99 percentiles), while the $D_{\text {me }}$ ranges from $82 \mu \mathrm{m}$ to $665 \mu \mathrm{m}$. The retrieved IWP error bars (one sigma in $\ln$ (IWP)) tend to be smaller for larger IWP and range from 0.21 to 1.8 . The high altitude cirrus around $15.2 \mathrm{~h}$ is retrieved due to the small brightness temperature depressions at 640 and $874 \mathrm{GHz}$, as no depression is seen at $220 \mathrm{GHz}$.

Figures 9 and 10 compare retrievals for scattering tables with three particle shapes and four shapes. Including solid ice hail (4 shapes) substantially reduces the minimum $\chi^{2}$ (better agreement with the CoSSIR observations) in high IWP regions. Using four shapes also results in more consistent retrieved shape mixing fractions from 13.8 to $13.9 \mathrm{~h}$, where high hail fractions are retrieved. The four shape retrievals have smaller IWP in the high IWP regions than the three shape retrievals, presumably because solid ice spheres are more efficient at microwave scattering. Even with the addition of the "hail" particle shape, the high IWP regions still have $\chi^{2}$ that is too high. Perhaps further refinement of the scattering particle shape models could improve the fit to the CoSSIR data. Figures 11 and 12 show that the minimum $\chi^{2}$ for two other flights seldom is greater than the $99 \%$ significance level. The $\chi^{2}$ bursts on 8 August are almost 


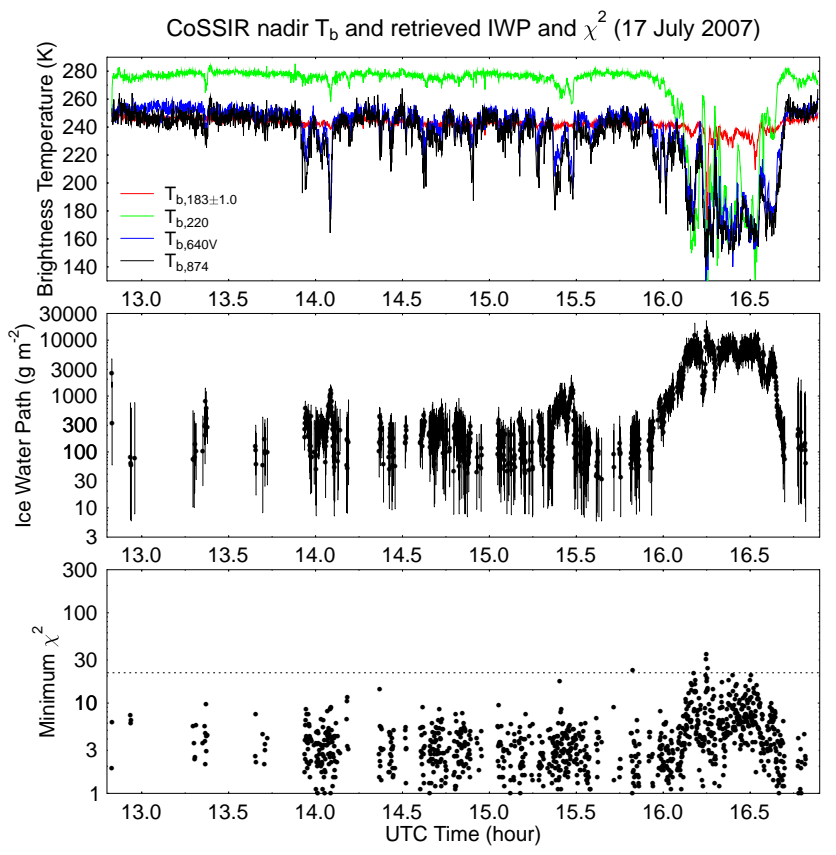

Fig. 11. CoSSIR brightness temperatures from 17 July for four channels (top panel). Retrieved ice water path with error bars of cloudy pixels (middle panel). Minimum $\chi^{2}$ (bottom panel); the dotted line at $\chi^{2}$ of 21.7 is the $99 \%$ significance level for 9 channels.

all associated with obvious "noise" on the $874 \mathrm{GHz}$ channel (e.g., spikes at $874 \mathrm{GHz}$ with no change at $640 \mathrm{GHz}$, though this is difficult to see in Fig. 12 without an expanded time scale).

Retrievals of ice water path and IWP error from Monte Carlo integration (MCI) and the optimization/local Gaussian method are compared in Fig. 13. The computer processing time for these nadir pixels is $0.21 \mathrm{~s}$ per pixel for the MCI method and $2.32 \mathrm{~s}$ per pixel for the optimization method. There are only optimization points for the largest IWP pixels where the MCI method does not have enough matching cases in the database for a retrieval. There are differences in the IWP retrievals between the two methods, but there is good agreement except for clear pixels. In clear sky the optimization sometimes retrieves an IWP of zero (not seen on the $\log$ scale) and sometimes retrieves a significantly nonzero value. When optimization retrieves an IWP of zero, the retrieved ln (IWP) error is very large, but, when the method retrieves a larger IWP for clear sky, the ln (IWP) error is between 0.5 and 2 (factors of 1.6 to 7.4 in IWP). There are substantial differences between the local Gaussian approximation retrievals of $\ln$ (IWP) error and the more reliable MCI retrievals. To quantify this, for the 249 pixels (out of 568 cloudy pixels) with MCI retrieved IWP $>100 \mathrm{~g} \mathrm{~m}^{-2}$, the difference in ln (IWP) error (local Gaussian - MCI) has a mean of 0.044 and a standard deviation of 0.224 . For comparison the MCI ln (IWP) error for these pixels has a mean of 0.572 and a standard deviation of 0.277 . To see if MCI

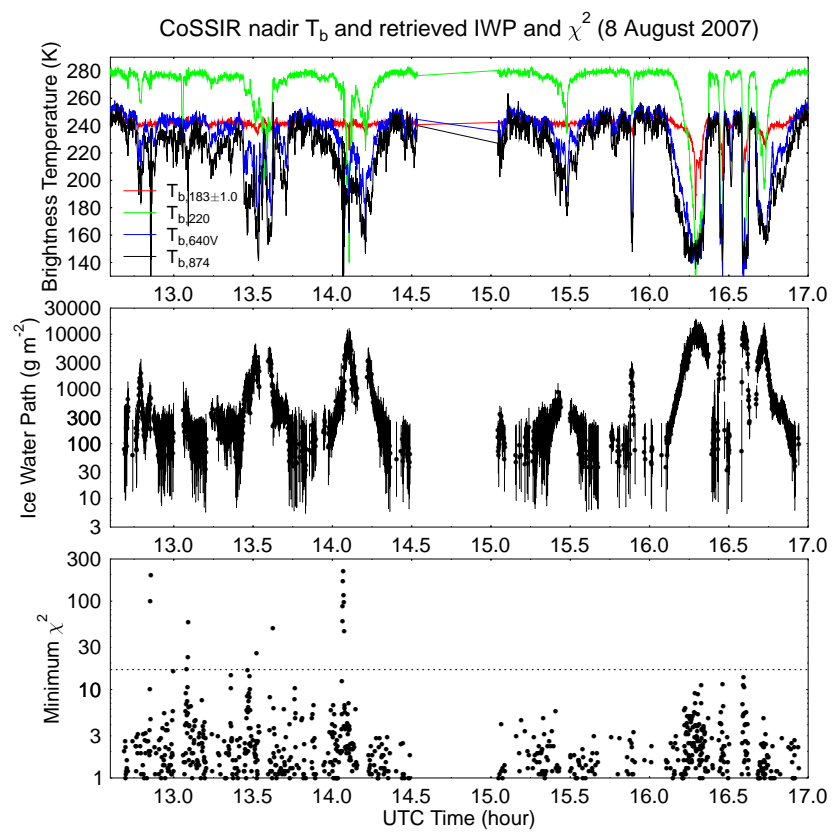

Fig. 12. CoSSIR brightness temperatures from 8 August for four channels (top panel). Retrieved ice water path with error bars of cloudy pixels (middle panel). Minimum $\chi^{2}$ (bottom panel); the dotted line at $\chi^{2}$ of 16.8 is the $99 \%$ significance level for 6 channels.

does indeed provide a more accurate IWP error than the local Gaussian approximation, the Markov chain Monte Carlo (MCMC) method is used for 24 pixels. The MCMC solution method is run with 200000 iterations (MC points), a burn in fraction of 0.5 , a final relaxation parameter of $10^{-5}$, and a relaxation parameter power of 0.3 . The computer time for the MCMC method is 1720 s per pixel. Figure 14 shows the comparison for the three solution methods. MCMC and MCI retrievals of IWP usually agree very well, while for some pixels the optimization is substantially different (though within the error bars). For the ln (IWP) errors, there is usually good agreement between MCI and MCMC and worse agreement with the local Gaussian approximation. The median absolute difference in $\ln$ (IWP) error for local Gaussian-MCI is 0.178 , for local Gaussian-MCMC is 0.133 , and for MCI-MCMC is 0.043 .

Validation of the CoSSIR ice cloud retrievals is made here by comparing $94 \mathrm{GHz}$ vertically integrated backscattering and radar reflectivity profiles retrieved from CoSSIR data with those from the Cloud Radar System (Li et al., 2004). The $94 \mathrm{GHz}$ backscattering from the CRS and CoSSIR retrievals is integrated from 5 to $15 \mathrm{~km}$. The integrated backscattering has units of $\mathrm{sr}^{-1}$, but the MCI retrieval and presentation are in $\mathrm{dB}\left(\mathrm{sr}^{-1}\right)$. Figure 15 shows the integrated backscattering comparison for the 19 July flight. The CoSSIR retrieved integrated backscattering agrees with that from CRS consistent with the retrieved 1- $\sigma$ error bars, and there is little apparent bias. Figure 16 shows the integrated 


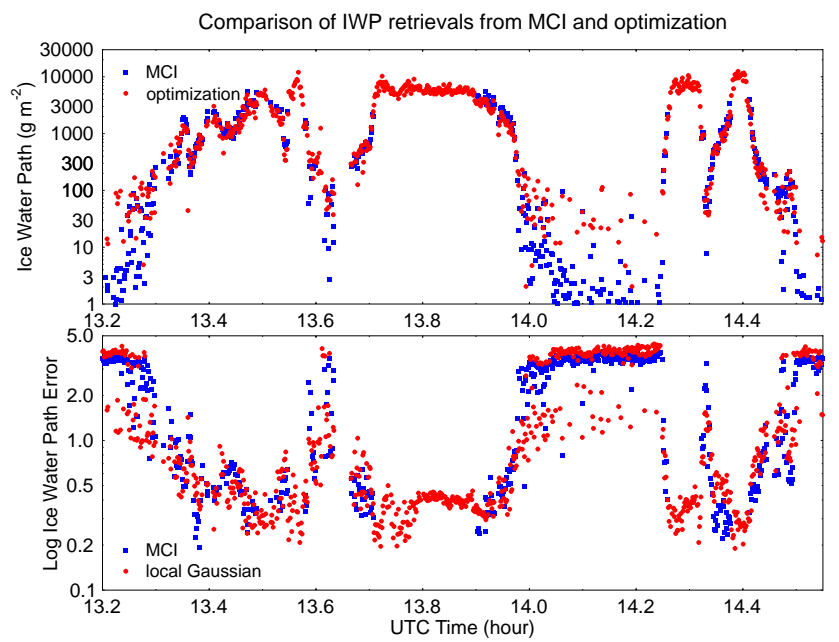

Fig. 13. Ice water path retrieved from CoSSIR on 19 July 2007 using Monte Carlo integration and optimization (top panel). ln (IWP) error retrieved using Monte Carlo integration and the local Gaussian approximation (bottom panel).

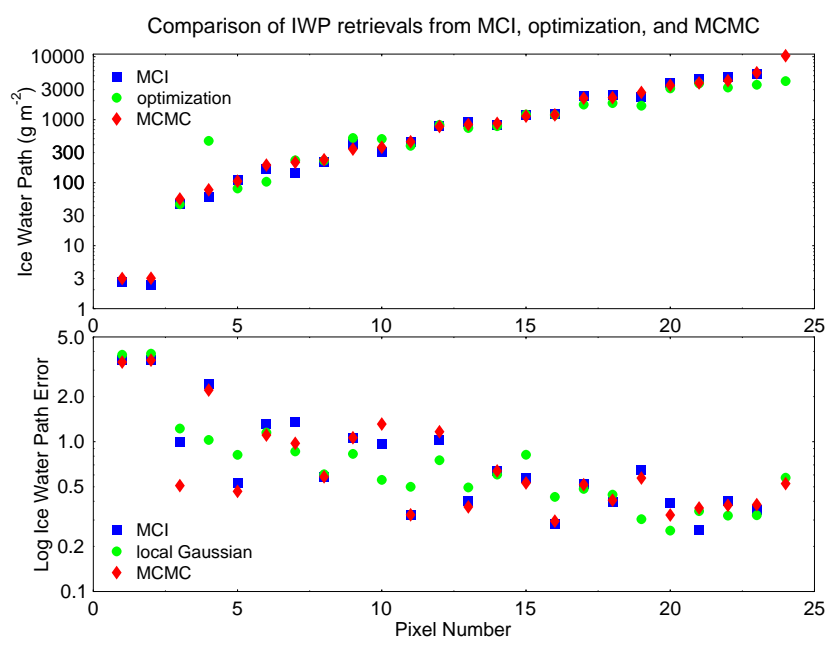

Fig. 14. Ice water path retrieved using Monte Carlo integration, optimization, and Markov chain Monte Carlo (top panel) for 24 selected pixels (sorted in order of MCMC IWP). $\ln$ (IWP) error retrieved using MCI, local Gaussian approximation, and MCMC methods (bottom panel).

backscattering comparison for all three flights. Again, the agreement is quite good over a factor of $1000(30 \mathrm{~dB})$. The 17 July and 8 August flights show a tendency for the greatest integrated backscattering to be a little less than the CRS values. Table 1 gives statistics of the integrated backscattering comparison for all the cloudy pixels. The rms difference is around $3 \mathrm{~dB}$, and the linear correlation is between 0.94 and 0.96. The reduced $\chi^{2}$ shows that the CoSSIR retrieved error bars are reasonably correct, considering that errors in CRS reflectivity or the volume mismatch between the two sensors are not included.

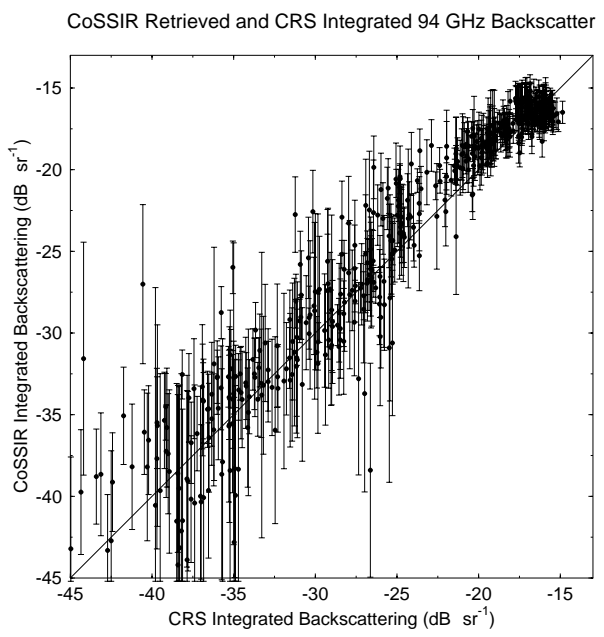

Fig. 15. A comparison of CoSSIR retrieved and Cloud Radar System integrated $94 \mathrm{GHz}$ backscattering for the 19 July flight.

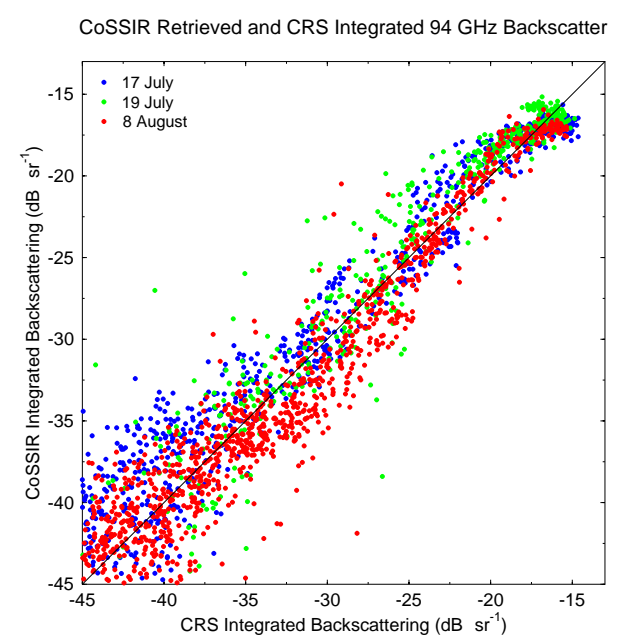

Fig. 16. A comparison of CoSSIR retrieved and Cloud Radar System integrated $94 \mathrm{GHz}$ backscattering for all three flights.

The ability of the algorithm to retrieve profiles is shown by comparing CoSSIR retrieved and CRS $94 \mathrm{GHz}$ reflectivity profiles in Fig. 17. The CoSSIR MCI integration retrievals are done in dBZ. There is clearly some ability of CoSSIR to retrieve vertical profile information. The high altitude strong echoes around 13.55 and $13.72 \mathrm{~h}$ and the general shape of the anvil echo between 13.75 and $13.95 \mathrm{~h}$ are seen in the CoSSIR retrieved profiles. The lower altitude ice reflectivity structure is retrieved poorly, which is not surprising given that water vapor blocks the higher-frequency channels seeing the midtroposphere. The higher altitude structure is retrieved well by CoSSIR when the reflectivity is high enough. More quantitatively for this flight, Fig. 18 shows the rms difference between CoSSIR retrieved and CRS reflectivity as a function of height. The CoSSIR retrieved and CRS reflectivity are averaged (in linear space, not dBZ) to thicker layers, because 
CRS and CoSSIR Retrieved 94 GHz Reflectivity (19 July 2007)

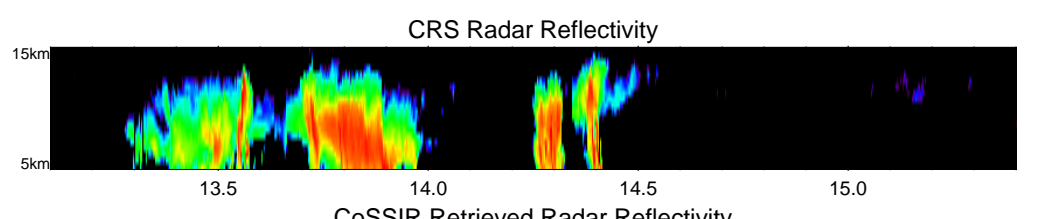

CoSSIR Retrieved Radar Reflectivity
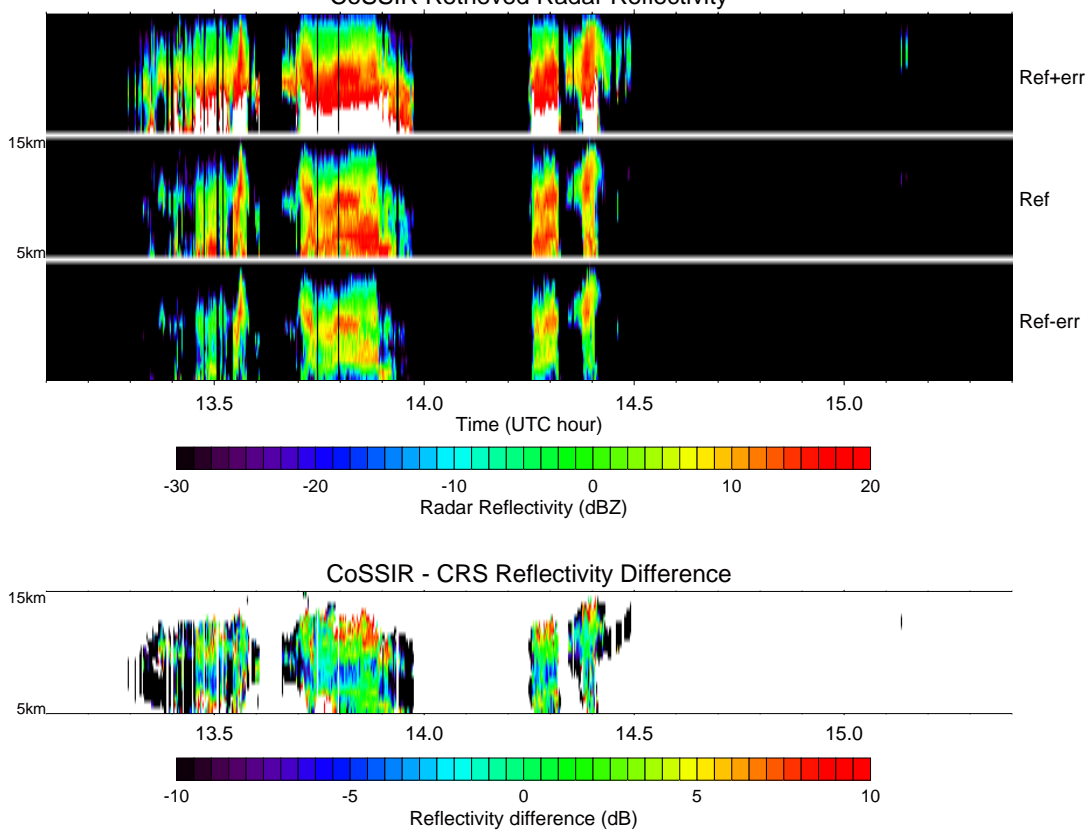

Fig. 17. A comparison of Cloud Radar System and CoSSIR retrieved $94 \mathrm{GHz}$ radar reflectivity profiles for CoSSIR determined cloudy columns. The 1- $\sigma$ error range of the retrieved reflectivity profiles is shown with the images above and below the CoSSIR retrieved profiles. In the difference plot at the bottom, pixels with CRS reflectivity below its noise level or non-cloudy CoSSIR retrievals are white.

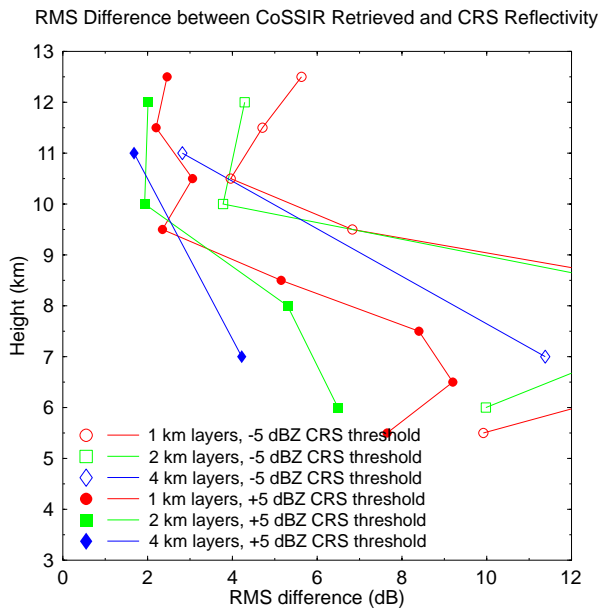

Fig. 18. RMS differences between CoSSIR retrieved and CRS $94 \mathrm{GHz}$ reflectivity as a function of height. The six curves are for different averaging layer thickness and minimum CRS reflectivity threshold. The marker is plotted at the center of each layer.
Table 1. Statistics on the comparison of the Cloud Radar System and CoSSIR retrieved vertically integrated $94 \mathrm{GHz}$ backscattering. The four statistics are the rms difference $(\mathrm{dB})$, the bias or mean difference $(\mathrm{dB})$, the reduced $\chi^{2}$ using the retrieved error bars, and the linear correlation.

\begin{tabular}{lrrrc}
\hline Flight & rms & bias & $\chi^{2} / N$ & corr \\
\hline 17 July & 3.29 & 1.25 & 1.63 & 0.962 \\
19 July & 2.70 & 0.80 & 1.55 & 0.962 \\
8 August & 3.02 & -0.19 & 0.89 & 0.944 \\
\hline
\end{tabular}

the CoSSIR retrieved profiles have coarse vertical resolution. Since the comparison is made in $\mathrm{dBZ}$, it is necessary to compare only those averaging layers with CRS reflectivity above a threshold. Figure 18 shows the CoSSIR retrieval error for $1 \mathrm{~km}, 2 \mathrm{~km}$, and $4 \mathrm{~km}$ thick layers and for layer average minimum CRS reflectivity of $-5 \mathrm{dBZ}$ and $+5 \mathrm{dBZ}$. The CoSSIR retrieval accuracy improves with thicker layers and larger CRS reflectivity thresholds. For $2 \mathrm{~km}$ layers with CRS reflectivity above $5 \mathrm{dBZ}$, the CoSSIR-CRS rms reflectivity difference is about $2.0 \mathrm{~dB}$ from 9 to $13 \mathrm{~km}$, but increases to between 5.3 and $6.5 \mathrm{~dB}$ from 5 to $9 \mathrm{~km}$. 
CoSSIR Retrieved IWC and $D_{m e}$ Profiles (19 July 2007)
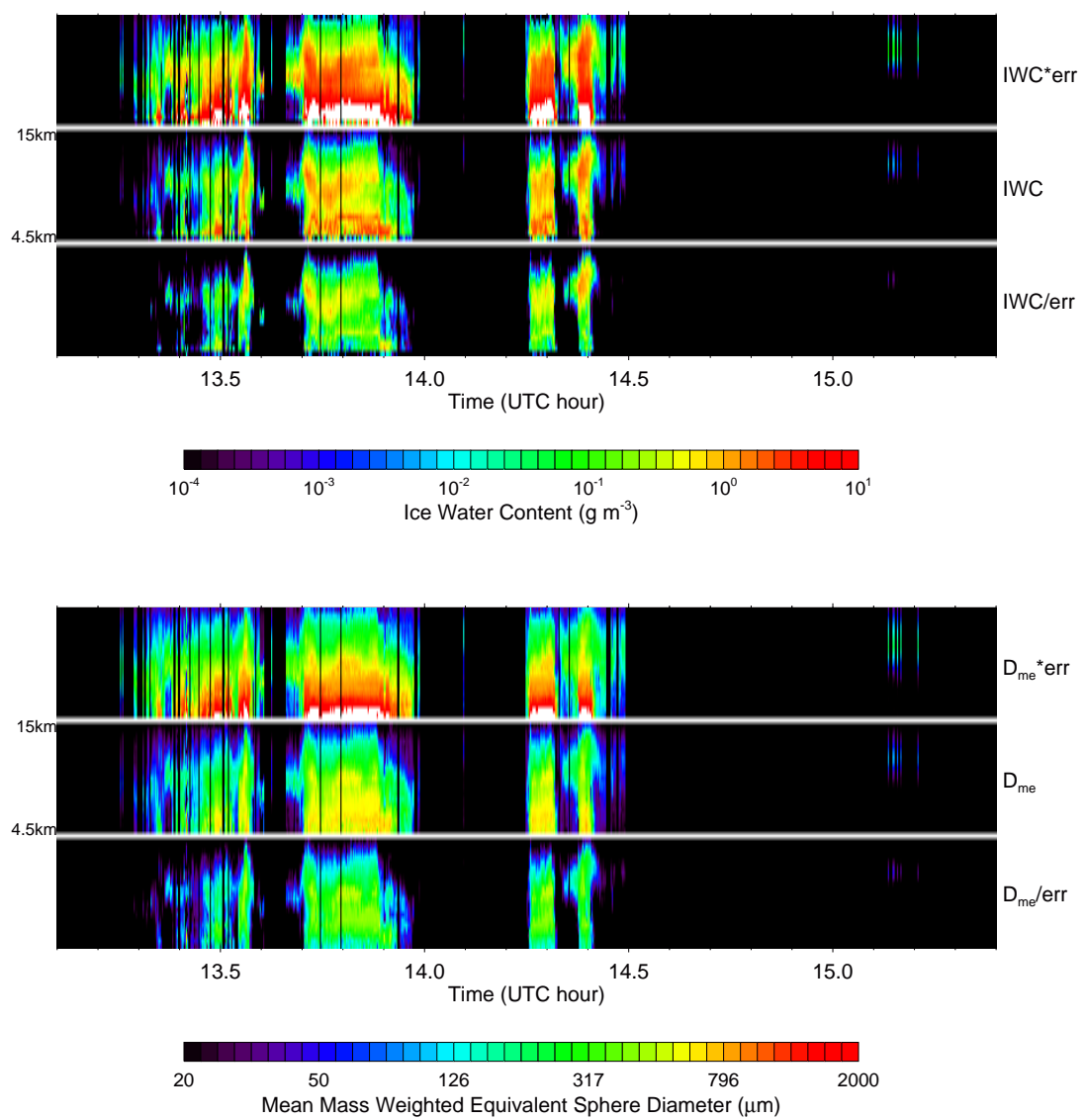

Fig. 19. Ice water content and $D_{\text {me }}$ profiles retrieved from nadir CoSSIR data. For each variable the middle time-height cross section is the retrieval, and the upper and lower images show the one standard deviation error range.

Figures 19 to 21 show time-height images of retrieved profiles of ice water content and $D_{\text {me }}$ from the Bayesian profile retrieval algorithm for inputs of CoSSIR alone, CRS alone, and the combination of CoSSIR and CRS data. Considering the large difference between the passive CoSSIR and active CRS, the agreement is quite good, certainly within the error range. The CoSSIR and CRS combined retrievals have a smaller error range, though the profiles are less continuous in time, which might be due to mismatched fields of view.

Figure 22 shows time-height images of relative humidity retrievals and error range made with nadir CoSSIR data. The relative humidity in deep ice cloud regions from about $3 \mathrm{~km}$ to $8 \mathrm{~km}$ is usually exactly 1.0 when retrieved using the optimization (i.e., the most probable posterior value). Since the retrieved error is symmetric, this leads to an unphysical upper error range above 1.0. It is possible that the a priori relationship between relative humidity and $\ln$ (IWC) is too strong. Outside of thick ice cloud, there is real humidity structure retrieved with the CoSSIR 183 and $380 \mathrm{GHz}$ channels. No validation of the relative humidity retrievals is shown.
Although CoSSIR is a scanning radiometer, the results shown to this point have been from the (noisier) nadir brightness temperature data, which allow direct comparison with the Cloud Radar System data and facilitate visualization of retrieved profiles. Figure 23 shows IWP and $D_{\text {me }}$ retrievals for CoSSIR forward conical scans. The retrievals are visualized by showing the CoSSIR beams as ellipses $(1.25 \mathrm{~km}$ by $1.75 \mathrm{~km}$ ) projected at $5 \mathrm{~km}$ altitude in a latitude-longitude map projection. Significant ice hydrometeor structure can be seen across the swath width of about $36 \mathrm{~km}$ (at $5 \mathrm{~km}$ altitude) along the flight track over two convective systems. The IWP and $D_{\text {me }}$ maps have somewhat different patterns, showing that IWP and $D_{\text {me }}$ are partially independent.

\section{Conclusions}

This article describes a Bayesian algorithm to retrieve ice water content, ice particle size ( $\left.D_{\mathrm{me}}\right)$, and relative humidity profiles from millimeter-wave and submillimeter-wave brightness temperatures. The prior pdf of cumulative distribution functions (CDFs) and EOFs for seven parameters and many 

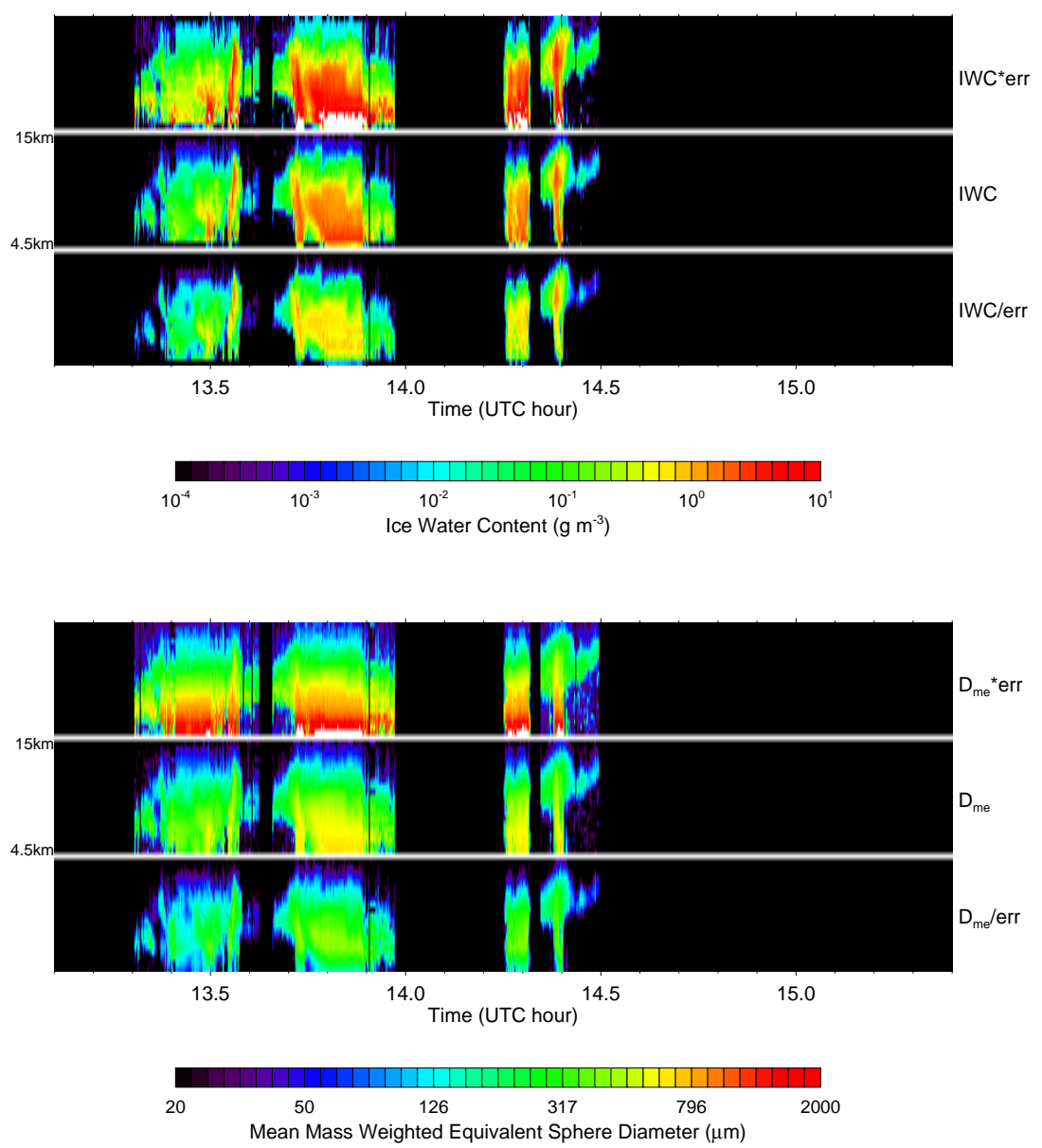

Fig. 20. Ice water content and $D_{\text {me }}$ profiles retrieved from nadir Cloud Radar System data.

layers is derived from the CloudSat radar reflectivity, lidar cloud fraction and ECMWF temperature and humidity profiles combined with three cloud microphysical pdfs obtained from in situ cloud probes. Retrievals from the Bayesian posterior pdf are obtained with Monte Carlo integration (MCI) and, when there are too few matching database cases, optimization to maximize the posterior pdf. Summaries of the algorithm are provided in Sect. 2 and in the flowchart of Fig. 1.

Expressing the Bayesian prior pdf in terms of CDFs allows for arbitrary (non-Gaussian) distributions, while the EOFs provide crucial correlations between the temperature, humidity, and the five hydrometeor parameters at all levels in the profile. The explicit prior function allows the Monte Carlo integration to be performed with any number of columns, and thus generalizes the information in the CloudSat columns used to make the prior pdf. Formulating the a priori information in the forward function with the radiative transfer (through a control variable transform) allows the use of the optimal estimation framework for a non-Gaussian prior. The hybrid solution method uses the highly efficient MCI method, but supplements it with optimization when there are not enough MCI database cases to match the observations. A poor match to observations after optimization (high $\chi^{2}$ ) indicates something is wrong with the observation vector or with the a priori or radiative transfer model. The advantage of using CloudSat associated data for a major part of the a priori information is that they provide rich information on vertical ice cloud structure anywhere around the world. Basing the microphysical pdfs on in situ data provides an objective method of introducing required additional a priori information.

A disadvantage of using cloud microphysical probability distributions obtained from in situ data is that they must be prepared for each region/season and can suffer from limitations of particular cloud probes and aircraft sampling. While the CDFs contain the complete single point statistical information, the EOFs, which are based on a correlation matrix, have only limited information about all higher-order statistics. Since this algorithm is primarily about retrieving ice hydrometeors from high-frequency microwave radiometer data, 
CoSSIR \& CRS Retrieved IWC and $D_{\text {me }}$ Profiles (19 July 2007)
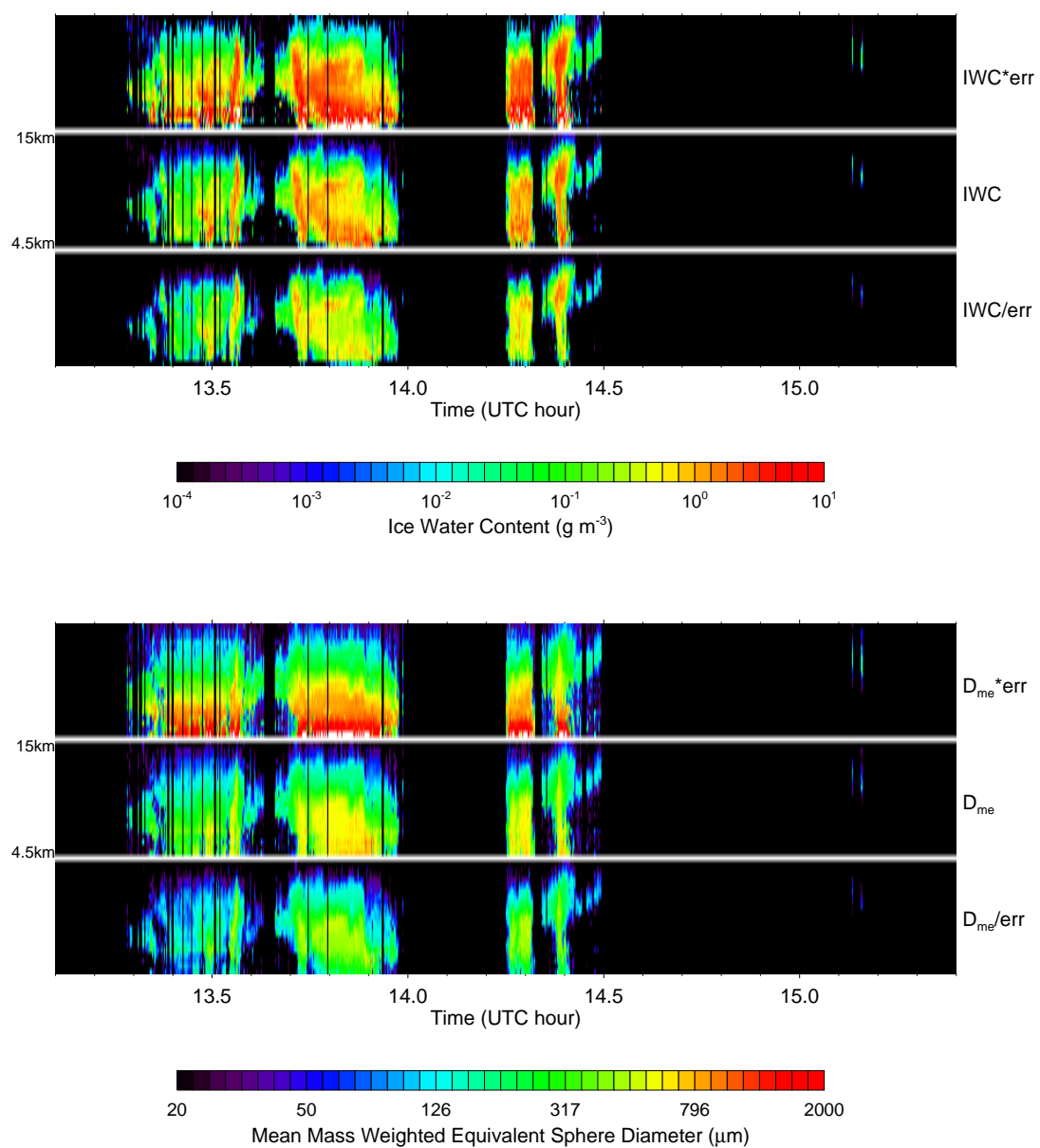

Fig. 21. Ice water content and $D_{\text {me }}$ profiles retrieved from nadir CoSSIR and Cloud Radar System data.

it mostly ignores clouds below the melting level. There are no boundary layer clouds, for example, though the CDF/EOF framework includes profiles of liquid water content and particle size, so low level clouds could be included. The ice particle melting model described in Appendix B4 is very simplified in that it assumes no updrafts and ignores evaporation, condensation, collection, and drop shedding. In addition, no raindrop microphysical pdfs are used, since the melting particle microphysics is based on the ice microphysics at the melting level. The significant limitations of the radiative transfer modeling are the common assumptions of randomly oriented particles and one-dimensional transfer.

Example retrieval results are mainly from CoSSIR data obtained on 19 July 2007 during TC4. Nadir retrievals are shown of the probability of ice clouds, and (with error bars) ice water path, $D_{\text {me }}$, and median IWP height. A comparison of the minimum $\chi^{2}$ and shape mixing fractions between retrievals including and excluding hail in the scattering tables indicated that, in some convective regions, solid ice hail was needed in addition to sphere aggregates (graupel) and snowflake aggregates. A comparison of the MCI and optimization methods showed that the retrieved IWP usually agreed well except for clear pixels. The agreement between MCI and optimization for the retrieved $\ln$ (IWP) error was poorer, likely because the local Gaussian assumption used for retrieving uncertainties with optimization was violated. The Markov chain Monte Carlo retrievals for two dozen pixels agree significantly better with the MCI method than the optimization/local Gaussian approximation method.

The ice particle retrieval results are validated by comparing vertically integrated $94 \mathrm{GHz}$ backscattering retrieved by CoSSIR with those measured by the Cloud Radar System on the same ER-2 aircraft. On three flights the rms difference between CoSSIR retrieved and CRS integrated backscattering for cloudy pixels is around $3 \mathrm{~dB}$ (over a $30 \mathrm{~dB}$ range) with a linear correlation of about 0.95 . The integrated backscattering agreement is consistent with the retrieved error bars and is better for larger values. A comparison is shown between CoSSIR retrieved and CRS radar reflectivity profiles. The error bars on the retrieved profiles are much larger than for the 
CoSSIR Retrieved Relative Humidity (19 July 2007)

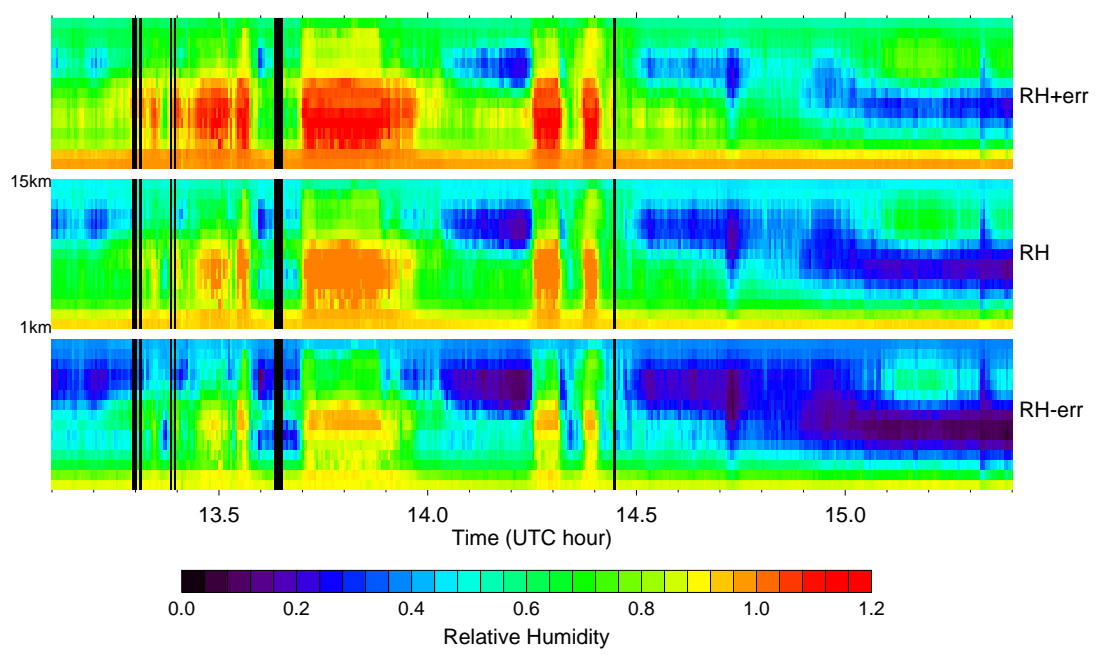

Fig. 22. Relative humidity profiles retrieved from nadir CoSSIR data from 1 to $15 \mathrm{~km}$ at $1 \mathrm{~km}$ resolution.
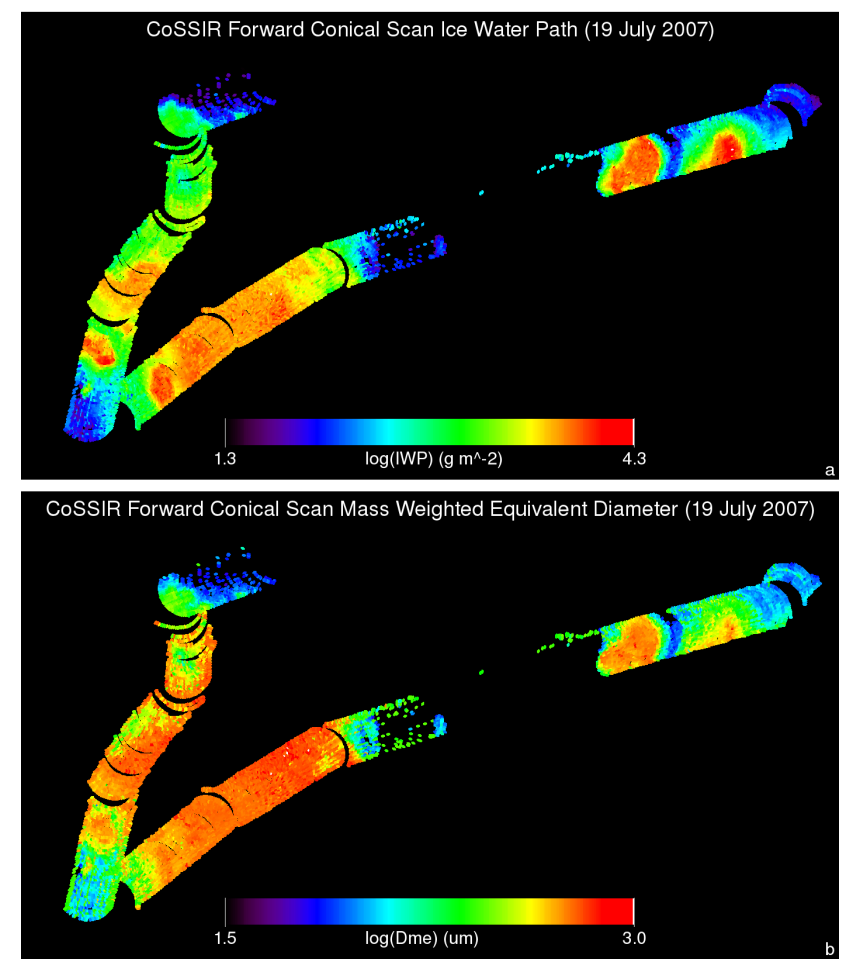

Fig. 23. Ice water path (a) and $D_{\text {me }}$ (b) of cloudy pixels retrieved from CoSSIR forward conical scans. The log color scale for IWP is from 20 to $20000 \mathrm{~g} \mathrm{~m}^{-3}$, while the $\log$ color scale for $D_{\mathrm{me}}$ is from 30 to $1000 \mu \mathrm{m}$. Only pixels with an ER-2 roll angle $<5^{\circ}$ are used. The pixels are projected to $5 \mathrm{~km}$ altitude. The longitude range is $90.2^{\circ} \mathrm{W}$ to $94.6^{\circ} \mathrm{W}$, and the latitude range is from $6.8^{\circ} \mathrm{N}$ to $10.0^{\circ} \mathrm{N}$. The retrievals are from 13.2 to $14.5 \mathrm{~h} \mathrm{UTC}$ on 19 July 2007. vertical integral, of course, but there is clearly some ability to retrieve profile information. Where the ice particle scattering signal is strong enough and at higher altitudes $(>9 \mathrm{~km})$, CoSSIR retrieved reflectivity agrees well with the CRS reflectivity (rms differences $\leq 3 \mathrm{dBZ}$ ). Nadir retrievals of IWC and $D_{\text {me }}$ using the Bayesian profile retrieval algorithm with CoSSIR data alone, CRS data alone, and the combination of CoSSIR and CRS data are shown for comparison. CoSSIR retrievals of IWP and $D_{\text {me }}$ for the forward conical scan in map view show interesting horizontal structure.

The ice cloud profile retrieval algorithm described here could be extended in a number of ways in the future. The code currently handles thermal emission and radar observables. No code changes are required to input infrared radiances in addition to microwave brightness temperatures, though particle scattering tables and K-distribution files would be needed for the infrared channels. Since the SHDOMPPDA model also performs solar radiative transfer, only minor changes would be required for the addition of visible radiances. Including infrared and visible radiances would likely require the addition of low-level liquid cloud to the prior pdf. The CloudSat radar is not sensitive enough to probe the full range of liquid clouds, but perhaps ground-based cloud radars could be combined with microphysical information from in situ cloud probes. Including lower-frequency microwave radiances would require a more correct formulation of the a priori raindrop information and an improved surface emissivity model, as well as the addition of low-level liquid clouds. A priori information about vertical rain structure could be provided by lower-frequency satellite radars. One area of improvement even for high-frequency microwave observations would be the inclusion of a priori ice particle shape information if relevant parameters, such as particle density, were available from in situ cloud probes. 
And finally, applying this retrieval algorithm to different ice cloud situations (e.g., wintertime synoptic systems) would require a different selection of ice particle shapes in the scattering tables and different microphysical input parameters.

\section{Appendix A}

\section{Analysis of TC4 in situ aircraft cloud microphysical data}

The cloud microphysical probability distribution inputs to the ice cloud profile retrieval algorithm are derived from in situ data from instruments flown in TC4. The key probability distribution is the multi-variate Gaussian distribution relating $T$ (temperature), $\ln \left(\right.$ IWC), $\ln \left(D_{\mathrm{me}}\right)$, and $D_{\mathrm{e}}$ dispersion for ice particles. The in situ ice particle size distributions are obtained from the two-dimensional stereo (2D-S) probe that flew on the DC-8 and WB-57 aircraft and the precipitation imaging probe (PIP) that flew on the DC-8. The 2D-S probe has true $10 \mu \mathrm{m}$ pixel resolution with 128 photodiode linear arrays and fast electronics (Lawson et al., 2006). The 2D-S has horizontal and vertical channels, though only the horizontal channel is used for the DC- 8 probe and the vertical channel for the WB-57 probe. The PIP probe has $100 \mu \mathrm{m}$ pixels and 64 photodiodes, and is used to sample larger ice particles. Data files for all the in situ instruments are obtained from the NASA Earth Science Project (ESPO) Office archive (http://espoarchive.nasa.gov/archive/ browse/tc4). The 2D-S and PIP files are available for the 11 DC-8 flights, and 2D-S files are available for the 4 WB-57 flights. While the 2D-S data files tabulate the size distributions up to a maximum dimension of around $3000 \mu \mathrm{m}$, the 2D-S and PIP number concentration spectra usually agree well for $D_{\max } \leq 1000 \mu \mathrm{m}$, but the 2D-S number concentration increasingly falls below the PIPs for larger sizes. So for the DC-8, the 2D-S data are used for $D_{\max }<1200 \mu \mathrm{m}$ and the PIP for $1200<D_{\max }<10000 \mu \mathrm{m}$, while for the WB-57 only the 2D-S data are used (larger particles are very rare at the WB-57 altitudes). The 1-s 2D-S samples are averaged to 5 -s samples to match the PIP.

As discussed in Sect. 2, the size distributions used in the ice cloud retrieval algorithm are based on particle mass or equivalent sphere diameter defined by $D_{\mathrm{e}}=\left[6 \mathrm{~m} /\left(\rho_{i} \pi\right)\right]^{1 / 3}$, where $m$ is the particle mass and $\rho_{i}=0.917 \mathrm{~g} \mathrm{~cm}^{-3}$ is the density of ice. The 2D-S and PIP probes measure shadowgraphs from which the maximum diameter $\left(D_{\max }\right)$ and projected area $(A)$ are derived (the PIP files only have $D_{\max }$ number concentration spectra). For consistency, power-law relationships between $D_{\mathrm{e}}$ and $A$ (for the 2D-S) and $D_{\mathrm{e}}$ and $D_{\max }$ (for the PIP) are obtained from three non-spherical ice particle shapes used in the scattering calculations (see Appendix B for details on these shapes). Figure A1 shows the $D_{\mathrm{e}}-A$ and $D_{\mathrm{e}}-D_{\max }$ scatter plots for the three particle shapes and the resulting fits. The power-law relation used for the 2D-S data is $D_{\mathrm{e}}=0.574 \mathrm{~A}^{0.429}$ (units of $\mathrm{mm}$ and $\mathrm{mm}^{2}$ ),
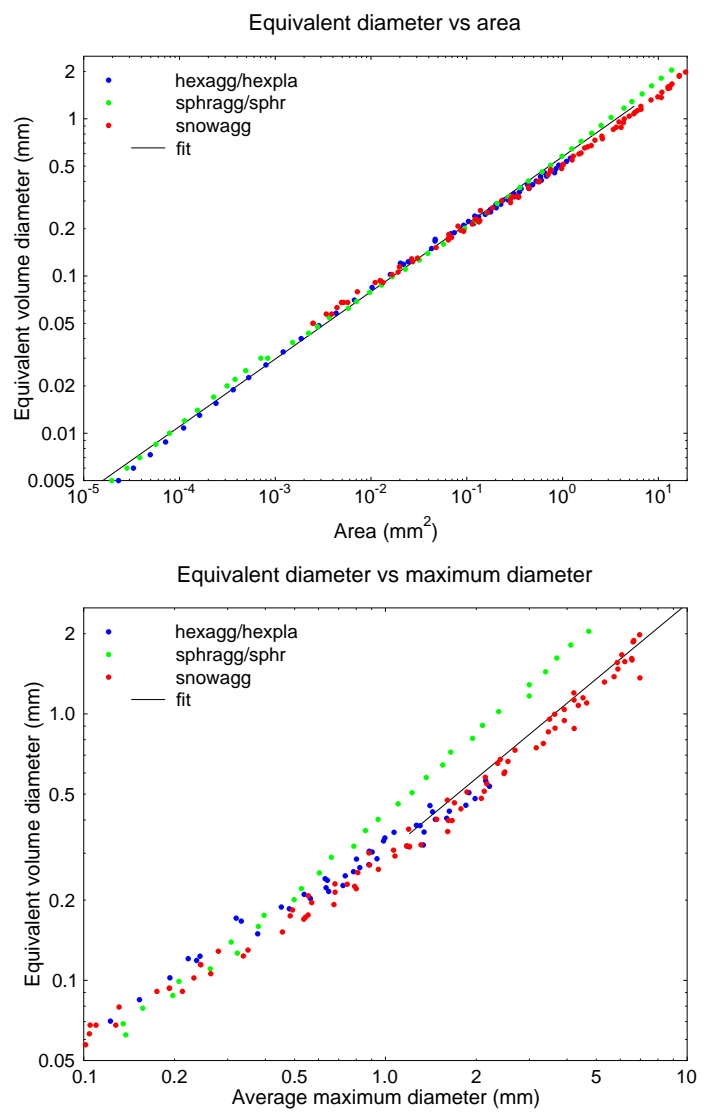

Fig. A1. The equivalent volume sphere diameter as a function of average projected area and average maximum diameter for randomly oriented particles used in the retrieval algorithm scattering tables. The power-law fits used for the 2D-S probe (top panel) and PIP (bottom panel) are also shown.

which is close to the one that SPEC used to process the 2D-S data $\left(D_{\mathrm{e}}=\min \left\{0.621 A^{0.406}, D_{\max }\right\}\right)$. The power-law fit used for the PIP data is $D_{\mathrm{e}}=0.299 D_{\max }^{0.938}$. The sphere aggregate shape has a substantially different curve from the other two shapes in the $D_{\mathrm{e}}-D_{\max }$ scatterplot, but it is not feasible in the retrieval algorithm to have separate fits for each shape.

The IWC, $D_{\text {me }}$, and $D_{\mathrm{e}}$ dispersion $\left(\sigma_{D_{\mathrm{e}}} / D_{\mathrm{me}}\right)$ are calculated for each size distribution from

$$
\begin{aligned}
& M_{j}=\frac{\rho_{i} \pi}{6} D_{\mathrm{e}}^{3} N_{j}, \quad \text { IWC }=\sum_{j} M_{j}, \\
& D_{\mathrm{me}}=\frac{\sum_{j} M_{j} D_{\mathrm{e}}}{\sum_{j} M_{j}}, \quad \sigma_{D_{\mathrm{e}}}^{2}=\frac{\sum_{j} M_{j} D_{\mathrm{e}}^{2}}{\sum_{j} M_{j}}-D_{\mathrm{me}}^{2},
\end{aligned}
$$

where $N_{j}$ is the number concentration in bin $j$ and $D_{\mathrm{e}}$ is obtained either from the projected area or $D_{\max }$ in bin $j$ using the power law relationships. The size distribution parameters are then merged with temperature from the Meteorological Measurement System (MMS) on either platform, and samples with temperatures above $270 \mathrm{~K}$ are removed. Histograms 

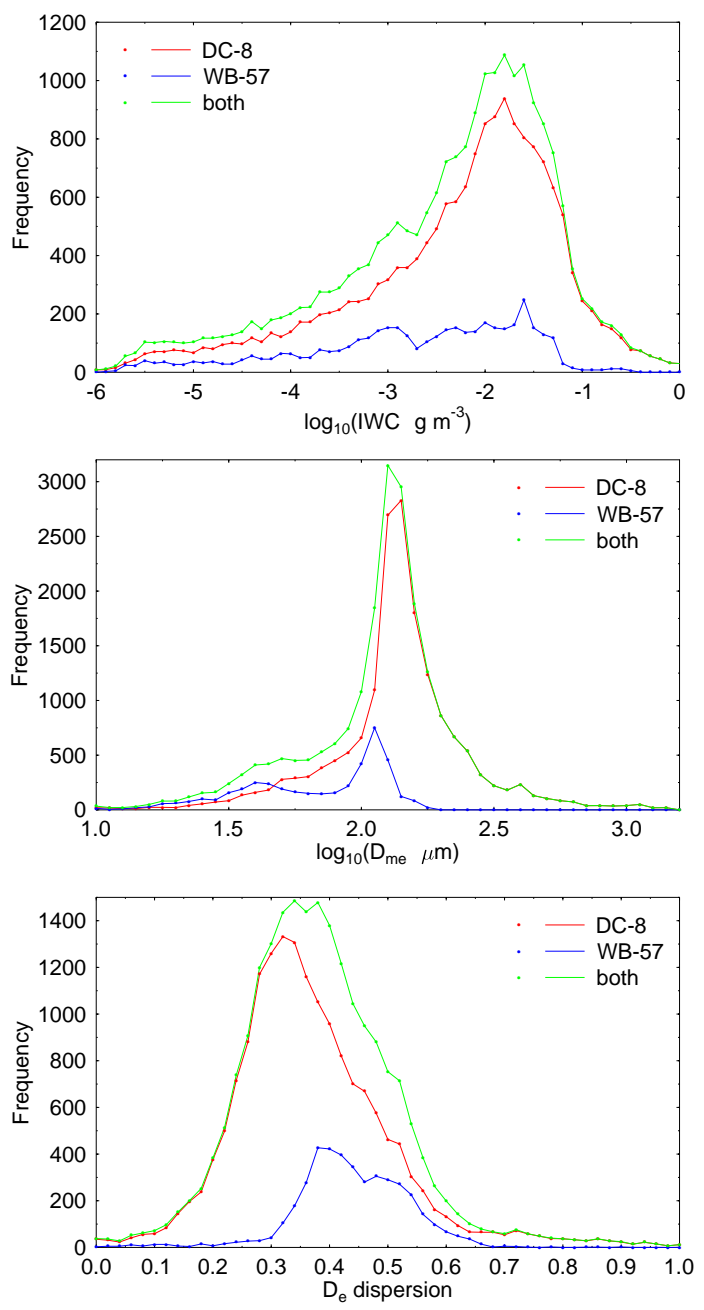

Fig. A2. Histograms of ice water content (IWC), IWC weighted mean equivalent sphere diameter $\left(D_{\mathrm{me}}\right)$, and equivalent diameter $\left(D_{\mathrm{e}}\right)$ dispersion from in situ cloud probes. The 2D-S \& PIP size distributions from the DC-8, the 2D-S size distributions from the WB57 , and both combined are shown.

of the resulting IWC, $D_{\mathrm{me}}$, and $D_{\mathrm{e}}$ dispersion for all DC8 flights, WB-57 flights, and both sets combined are shown in Fig. A2. The size distributions from both aircraft cover a rather wide range of values, but the WB-57 does not have the highest values of IWC and $D_{\text {me }}$, because it flew at colder temperatures and stayed out of the updrafts. There are much fewer samples for the WB-57 because it only flew on four flights. A scatterplot of $D_{\text {me }}$ versus temperature is shown in Fig. A3. There is a fairly strong correlation between the typical particle size as measured by $D_{\mathrm{me}}$ and temperature over the whole range of temperatures. The DC-8 ceiling limits the minimum temperature to about $215 \mathrm{~K}$, but the WB-57 extends the temperature to $193 \mathrm{~K}$. Figure A4 shows the relationship between $D_{\text {me }}$ and IWC for the size distributions from the two aircraft. There is clearly a correlation between $D_{\text {me }}$ and IWC, though the pattern is not simply bivariate log-normal.

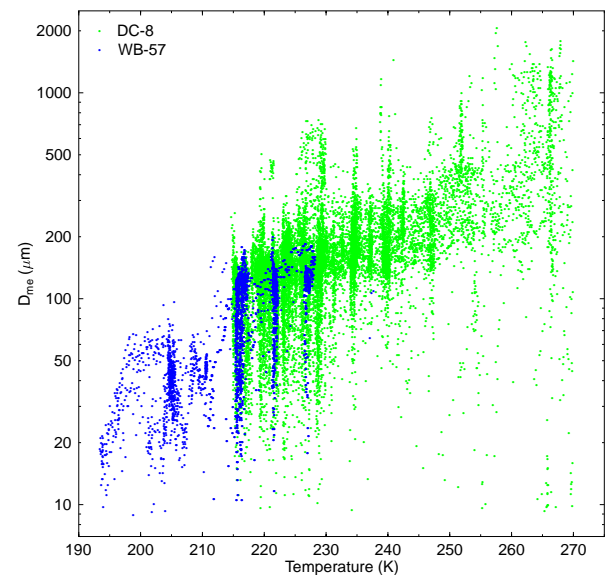

Fig. A3. Scatterplot of $D_{\text {me }}$ versus temperature for in situ size distributions from the DC-8 and from the WB-57. The clusters of samples at particular temperatures are due to extended flight segments at particular altitudes.

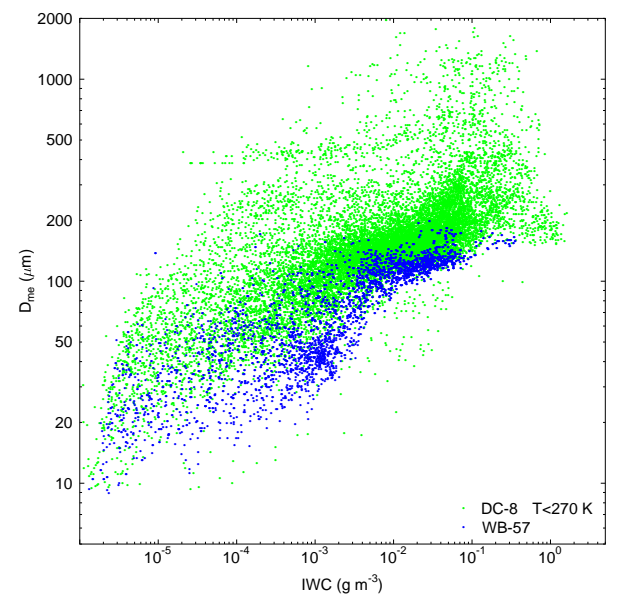

Fig. A4. Scatterplot of $D_{\text {me }}$ versus ice water content for in situ size distributions from the DC-8 (2D-S and PIP) and from the WB-57 (2D-S).

One difficulty with using aircraft microphysical data for ice cloud a priori information is that it is seldom completely representative of the natural distribution of cloud ice microphysics. The issues of the size ranges, to which different probes are sensitive, and of converting from 2-D optical probes to the mass related parameters have already been discussed. Another issue is that, during typical field campaigns, the aircraft sampling is seldom random and complete, partly due to aircraft limitations and partly due to the multiple objectives of the campaign. In the case of TC4, the aircraft sampling was constrained by the ceiling of the DC-8, the limited participation by the higher altitude WB-57, the need to avoid strong updrafts in convective cores, and the science objectives of sampling the outflow anvils (mostly colder than $230 \mathrm{~K})$. Thus, the ice microphysics scatter plots shown in 
Fig. A4 are certainly biased. About $75 \%$ of the data lie between temperatures of $215 \mathrm{~K}$ and $235 \mathrm{~K}$, and for this reason and others $50 \%$ of the $D_{\text {me }}$ lie between $100 \mu \mathrm{m}$ and $175 \mu \mathrm{m}$ (though the maximum $D_{\text {me }}$ is greater than $1600 \mu \mathrm{m}$ ).

Rather than use an empirical probability distribution of ice microphysics based on the 2D-S and PIP data, a multi-variate normal (Gaussian) distribution in temperature, $\ln$ (IWC), $\ln \left(D_{\mathrm{me}}\right)$, and $D_{\mathrm{e}}$ dispersion is used. This allows the microphysical pdf to be specified with only a few parameters, use the a priori idea that IWC and $D_{\text {me }}$ are approximately log-normal, generalize the microphysical data to some extent, and use the all important correlation between temperature, $D_{\text {me }}$, and IWC. Since the TC 4 microphysical dataset used here is decidedly non-Gaussian, one has to be careful about calculating the parameters of the equivalent multivariate Gaussian pdf. For example, in the 2D-S/PIP dataset $\ln$ (IWC) has a long negative tail representing very low IWC values. Using the traditional method to calculate the parameters of the Gaussian distribution ln (IWC) results in a large standard deviation, which leads to extremely large a priori IWC (e.g., $3 \sigma$ IWC at $273 \mathrm{~K}$ is $167 \mathrm{~g} \mathrm{~m}^{-3}$ ). Instead, two percentiles of the microphysical distribution are used to characterize the equivalent Gaussian distribution. For $\ln$ (IWC) the median and 95th percentile ( $\left.p_{1}=0.5, p_{2}=0.95\right)$ are used; for $\ln \left(D_{\mathrm{me}}\right) p_{1}=0.5, p_{2}=0.90$; for temperature $p_{1}=0.05$, $p_{2}=0.95 ;$ and for $D_{\mathrm{e}}$ dispersion $p_{1}=0.25, p_{2}=0.75$. The equivalent Gaussian correlations between the four parameters are obtained from rank correlations, which are nonparametric. As in Sect. 3, the cumulative distribution probability of each dimension is converted to a standard normal distribution value for each sample, and the correlation of these Gaussian variables is calculated using a correlation matrix. The resulting parameters of the ice microphysics Gaussian distribution are listed in Table A1. The table also lists some of the IWC and $D_{\text {me }}$ that the Gaussian distribution generates for certain given temperatures.

In moderate to strong updrafts, we expect supercooled liquid cloud droplets to occasionally coexist with ice particles. It is important to include supercooled cloud droplets in a microwave retrieval algorithm, because liquid droplets are much more absorbing than ice particles, and so potentially can significantly affect the upwelling brightness temperatures. To include appropriate a priori information on supercooled droplets, the relationship between liquid cloud water content (LWC) and mass weighted mean droplet diameter $\left(D_{\mathrm{me}}\right)$, and temperature and ice water content is sought. During TC4 there were no cloud probes that could accurately measure liquid cloud droplets in the presence of ice particles. The only cloud probe on the DC- 8 designed to count and size cloud droplets was the Cloud and Aerosol Spectrometer (CAS) (Baumgardner et al., 2001) from Droplet Measurement Technologies. The CAS has no way to distinguish between small ice particles and liquid droplets. Furthermore, Jensen et al. (2009) established that the CAS is highly susceptible to spurious high concentrations of small particles

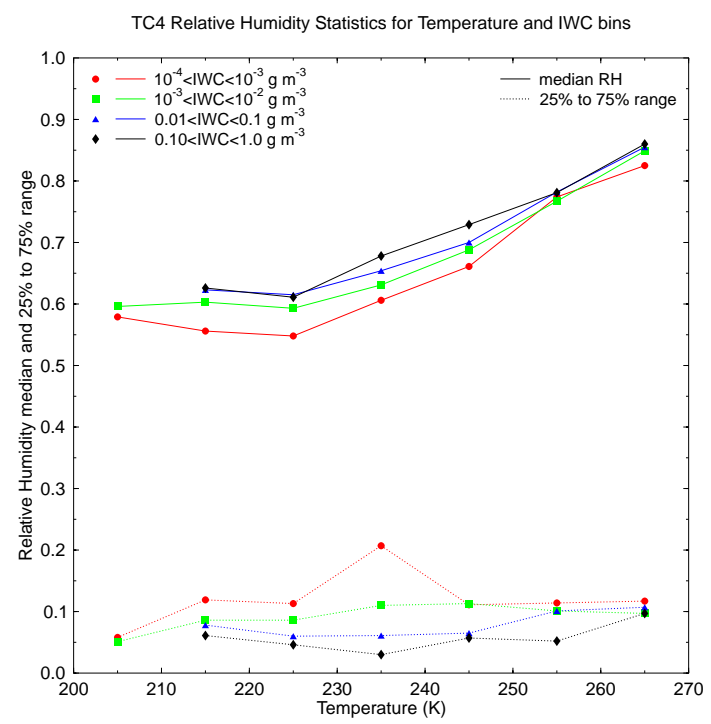

Fig. A5. Relative humidity median and distribution width (25\% to $75 \%$ range) as a function of temperature for four ice water content bins.

due to shattering of large ice crystals on the CAS inlet. Nevertheless, CAS data are used here with a variety of thresholds to reduce the ice particle contamination. The resulting liquid cloud droplet distributions are still highly suspect, and should be thought of more as a placeholder, in that it is better to have some a priori distribution of supercooled cloud droplets than assume none in the retrieval algorithm.

The CAS data are used for seven DC-8 flights for which it was working during TC4. The LWC and $D_{\text {me }}$ are calculated from the number concentration spectra, and then merged with the MMS temperature and the 2D-S/PIP IWC. Samples with temperature outside the range 240 to $273 \mathrm{~K}$, with $D_{\mathrm{e}}$ dispersion greater than 0.4 , with $D_{\text {me }}$ outside the range from 8 to $40 \mu \mathrm{m}$, or with IWC less than $10^{-6} \mathrm{~g} \mathrm{~m}^{-3}$ are removed. The CAS LWC is multiplied by a linear factor in temperature ( 0 at $240 \mathrm{~K}, 1$ at $273 \mathrm{~K}$ ) to attempt to distinguish between small ice crystals and cloud droplets. A multi-variate Gaussian distribution in temperature, $\ln$ (IWC), $\ln$ (LWC), and $\ln \left(D_{\text {me,liq }}\right)$ is fit to the data using the robust percentile method described for the ice microphysical distribution. The mean $\ln (\mathrm{LWC})$ is -6.24 (median supercooled LWC is $0.00195 \mathrm{~g} \mathrm{~m}^{-3}$ ), and the standard deviation of $\ln$ (LWC) is 2.29 (so 99th percentile LWC is $0.71 \mathrm{~g} \mathrm{~m}^{-3}$ ). The correlation between $\ln$ (IWC) (ice particles) and $\ln$ (LWC) (supercooled droplets) is 0.55 , and between temperature and $\ln$ (LWC) is 0.61 .

Due to the large-scale nature of the ECMWF fields, the relative humidity profiles do not have realistic correlation with cloud ice water content. During TC4 a diode laser hygrometer (DLH, Diskin et al., 2002) on the DC-8 and the JPL Laser Hygrometer (JLH) on the WB-57 accurately measured water vapor density in the presence of ice particles. The laser hygrometer data for 11 DC-8 flights and 4 WB-57 flights 
Table A1. Ice particle microphysical statistics defining the a priori Gaussian probability distribution.

\begin{tabular}{llllc}
\hline & Temperature $(\mathrm{K})$ & $\ln (\mathrm{IWC})\left(\mathrm{g} \mathrm{m}^{-3}\right)$ & $\ln \left(D_{\mathrm{me}}\right)(\mu \mathrm{m})$ & $D_{\mathrm{e}}$ dispersion \\
\hline Mean & 233.75 & -4.779 & 4.924 & 0.388 \\
Std dev & 11.44 & 1.609 & 0.469 & 0.118 \\
Correlations & $\rho_{T \text {-IWC }}=0.351$ & $\rho_{T \text { - }} D_{\text {me }}=0.664$ & $\rho_{\text {IWC- } D_{\text {me }}}=0.708$ & \\
& $\rho_{T \text {-disp }}=-0.205$ & $\rho_{\text {IWC-disp }}=0.113$ & $\rho_{D_{\text {me }} \text {-disp }}=-0.138$ & \\
\hline & Temperature $(\mathrm{K})$ & IWC $\left(\mathrm{g} \mathrm{m}^{-3}\right)$ & $D_{\text {me }}(\mu \mathrm{m})$ & \\
\hline+3 std dev & 273.2 & 7.34 & 1646.5 & \\
+0 std dev & 273.2 & 0.06 & 403.0 & \\
+3 std dev & 235.0 & 1.12 & 581.7 & \\
-3 std dev & 200.0 & $1.27 \times 10^{-5}$ & 13.4 & \\
\hline
\end{tabular}

are merged with MMS temperature and pressure, converted to relative humidity with respect to liquid, and merged with the 2D-S/PIP ice water content. To determine how the relative humidity depends on temperature and IWC, samples are grouped into $10 \mathrm{~K}$ bins of temperature and factor of 10 bins in IWC. The median relative humidity with the range from the 25 th to 75 th percentiles for the temperature and IWC bins is graphed in Fig. A5. The median relative humidity increases with temperature, slowly at first below $230 \mathrm{~K}$, and then more rapidly. The median relative humidity tends to increase with IWC, though not so much at the higher temperature bins (which have many fewer samples, though). The width of the relative humidity distribution has little temperature dependence but does decrease with increasing IWC.

A beta distribution of relative humidity is assumed with the mean and standard deviation depending on temperature (T) and IWC according to

$$
\begin{aligned}
& \mathrm{RH}_{\text {mean }}=a+b T+c T^{2}+\mathrm{d} \ln (\mathrm{IWC}) \\
& \mathrm{RH}_{\text {stddev }}=e+f \ln (I W C) .
\end{aligned}
$$

The coefficients are obtained from the relative humidity samples with temperature less than $270 \mathrm{~K}$ by minimizing the negative of the log-likelihood of the beta distribution pdf with the downhill simplex method. The resulting coefficients are $a=6.989, b=-0.0571, c=0.0001309$, $d=0.01417, e=0.03844$, and $f=-0.007965$, for $T$ in $\mathrm{K}$ and IWC in $\mathrm{g} \mathrm{m}^{-3}$.

\section{Appendix B}

\section{Particle shape models and preparation of scattering tables}

Particle shape is the most difficult part of ice cloud remote sensing, and that is also true for submillimeter-wave sensing. The longer wavelengths of cloud radars and millimeter-wave and submillimeter-wave radiometers are sensitive to larger particles and the broader features of particle shape, as compared with visible and infrared sensing. Here the word shape refers to a set of related ice particle shapes over a wide range of sizes, i.e., not one well-defined shape that is simply scaled with size. Although some ice crystal shapes and sizes are known to fall with a preferred orientation, the radiative transfer model used in the retrieval program assumes randomly oriented particles, and so that is assumed for the ice particle scattering calculations. In this work particle shapes are sought to model the microwave radiative properties of ice particles in tropical convective cores, stratiform regions, and cirrus anvils. The goal is not the impossible task of simulating all of the possible ice particle shapes in tropical convection, but to have a few types of realistic particle shapes that span the relevant properties of randomly oriented particles, such that mixtures of these particles can simulate the microwave radiative transfer of the the actual ensemble of particles found in nature.

\section{B1 Hexagonal plate and sphere aggregates}

As reported in Evans et al. (2005), Cloud Particle Imager pictures of ice particles in convective anvils sampled in CRYSTAL-FACE were mostly irregular particles, and often aggregates of spheres or hexagonal plates. When $10^{4}$ to $10^{5}$ spheres are aggregated, the sphere aggregates would appear to be a good model of graupel, which is an appropriate particle type for tropical convective cores. The $30 \mu \mathrm{m}$ diameter stochastic ice sphere aggregates modeled in Evans et al. (2005) are extended here on the high end to larger number of sphere monomers (up to 315000 ) and on the low end by ice spheres ranging from 2 to $30 \mu \mathrm{m}$ in diameter. The two sizes of hexagonal plate aggregates modeled in Evans et al. (2005) are extended here only on the low end with single hexagonal plates ranging in maximum diameter from 5 to $250 \mu \mathrm{m}$. The hexagonal plate aggregate and sphere aggregate shapes are illustrated in Fig. B1. The low density ice spheres modeled in Evans et al. (2005) are not used here because microwave scattering properties of equivalent spheres are a very poor approximation to accurate calculations of complex realistic shaped ice particles (e.g., Kulie et al., 2010). 


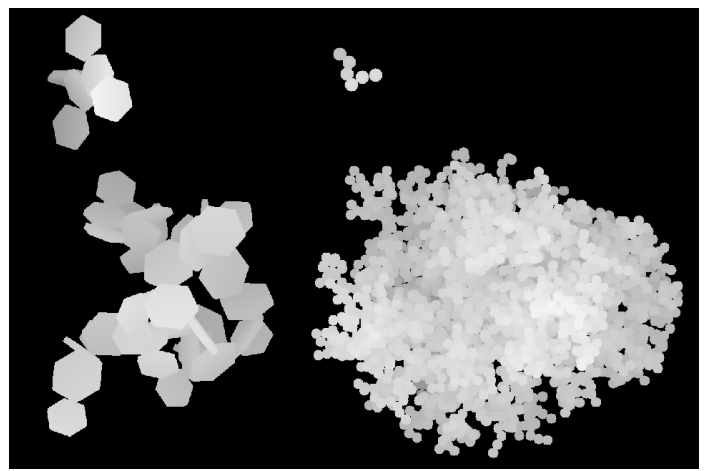

Fig. B1. Image showing two hexagonal plate aggregates (left) and two sphere aggregates (right). The plate aggregates are made of 6 thinner plates and 40 thicker plates. The sphere aggregates have 6 and 3600 spheres. The lighter shades indicate the closer parts of the particle, and the darker shades indicate the farther parts.

\section{B2 Snowflake aggregation model}

Graupel is one type of large ice particles associated with precipitation in convective systems, but a lower density particle type is needed to model snowflake aggregates associated with stratiform regions. Snowflake aggregates are modeled here with a physically based simulation of aggregation of twodimensional dendritic crystals. The 2-D dendritic crystals are generated with the semi-physical crystal depositional growth simulation code of Gravner and Griffeath (2008). There is no method for mapping their eight input parameters to temperature and vapor pressure, so many simulations with random parameters were performed and dendritic crystals selected subjectively. These 2-D dendrites are assigned a thickness depending on diameter from the Auer and Veal (1970) power-law formula for thickness of dendrites. Petty and Huang (2010) generated random aggregates of dendrites digitized from snow crystal microphotographs, which were then used for modeling lower frequency microwave scattering.

The 2-D dendrites are aggregated in a Monte Carlo physical simulation similar to Maruyama and Fujiyoshi (2005), who used sphere monomers. A large number of particles are introduced to a fixed volume with an initial gamma size distribution in equivalent volume diameter. The Monte Carlo aggregation method is based on Gillespie (1975). The collision rate is calculated for all pairs of particles using the relative fall speeds and the convex circumscribed area and perimeter of the particles. The Heymsfield and Westbrook (2010) parameterization for fall speeds is used. The particles are assumed to fall with a horizontal orientation. A pair of particles is randomly selected to collide according to the collision rate matrix. The centroids of the two particles are randomly offset within a convex polygon given by the convex hull of the faster falling particle expanded by the "radius" of the slower particle. The particle being collected is randomly rotated over all three axes. The two particles are then combined vertically until they overlap by some small fraction ( $5 \%$ used here) of the mass of the lower particle. There is no physical fitting together of the dendrite branches or breaking of the snowflakes. Each snowflake aggregate is represented by a list of the position, rotation matrices, and 3-D array of filled voxels of its dendrite components. The upper (collecting) and lower (collected) particles in a collision are rendered into a large 3-D array to determine if and at what offset the two particles join. After a collision, if a particle moment of inertia principal axis is more than a specified angle from vertical ( $2^{\circ}$ used here), the particle is rotated so that it is back in balance. New pristine crystals from the original gamma distribution may be stochastically created according to a vapor deposition mass rate. Aggregates that have fallen more than a specified distance, which tend to be the largest fastest falling ones, are removed from the simulation volume, but included in the output.

The snowflake aggregates used in the scattering tables are generated in one aggregation simulation. The input crystals to the simulation were 562 -D dendrites ranging in diameter from 100 to $2000 \mu \mathrm{m}$. The input dendrites have $2 \mu \mathrm{m}$ pixel size, but the voxel size in the aggregation simulation is $16 \mu \mathrm{m}$, and the output grid resolution used for scattering calculations is $32 \mu \mathrm{m}$. There are 3000 initial particles, made from multiple copies of the input 56 dendrites, having an ice water content of $0.1 \mathrm{~g} \mathrm{~m}^{-3}$ and an initial gamma distribution $D_{\text {me }}$ of $300 \mu \mathrm{m}$. The fall speed calculations are carried out at $400 \mathrm{mb}$ pressure and $-15^{\circ} \mathrm{C}$ (the temperature of maximum dendritic growth). The snowflakes are given $4000 \mathrm{~m}$ before falling out of the aggregation zone. The vapor mass deposition rate is $1.5 \times 10^{-4} \mathrm{~g} \mathrm{~m}^{-3} \mathrm{~s}^{-1}$, and the simulation time is $3 \mathrm{~h}$. The aggregation simulation ends with 1429 in cloud particles (with an IWC of $0.208 \mathrm{~g} \mathrm{~m}^{-3}$ ) and 56 fallen aggregates (with a melted precipitation depth of $5.85 \mathrm{~mm}$ ). Of these snowflakes 89 were chosen for the scattering calculations, ranging in equivalent diameter $\left(D_{\mathrm{e}}\right)$ from 51 to $1986 \mu \mathrm{m}$ and maximum diameter from 128 to $8884 \mu \mathrm{m}$. The goal of the selection was to choose three aggregates in each $0.5 \mathrm{~dB} D_{\mathrm{e}}$ bin with aspect ratios spaced from the median to the maximum or, if only unaggregated dendrites are in the size bin, to choose different dendrites. This approach results in a variety of snowflake aggregate morphologies covering a large range of particle sizes for making size distributions. Renderings of seven of the snowflakes are shown in Fig. B2.

\section{B3 DDA calculations for the scattering tables}

Results in Sect. 5 show that the radiative transfer modeling is a poor fit to the CoSSIR brightness temperatures in some high IWP situations with ice particle mixtures of the plate aggregates, sphere aggregates, and snow aggregates. The fits improved with the addition of large solid ice spheres meant to represent hail. Thus, most results presented are for scattering tables including four particle shapes (or types). 


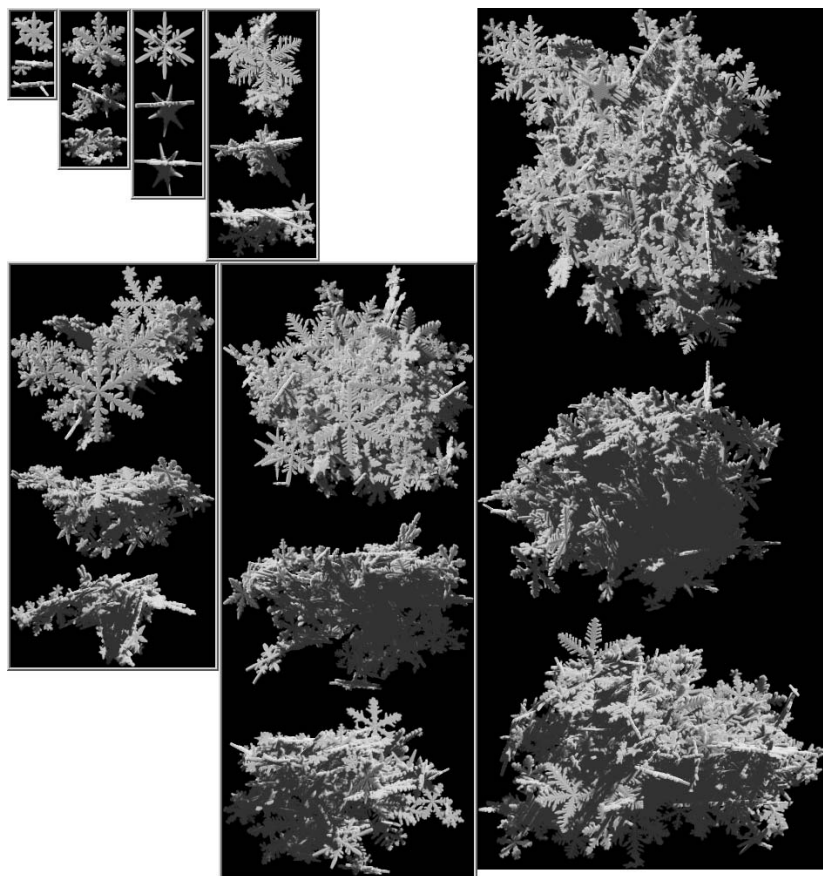

Fig. B2. Images showing seven snowflake aggregates, each from three perspectives: view of $\mathrm{X}-\mathrm{Y}$ plane (top panel), view of $\mathrm{X}-\mathrm{Z}$ plane (middle panel), and view of Y-Z plane (bottom panel). A solar pseudo-radiative transfer rendering is used; the solar illumination is from the upper left at $45^{\circ}$ zenith angle. The equivalent volume diameters of these aggregates are 254, 369, 321, 510, 854, 1315, and $1881 \mu \mathrm{m}$.

Except for ice spheres, the scattering calculations for randomly oriented ice particles are computed with the discrete dipole approximation (DDA). This method divides the particle up into many dipoles with the dipole size being small compared to the wavelength; generally the dipole size is $32 \mu \mathrm{m}$ or smaller. The dipole discretization of a particular particle is kept the same for all frequencies. The matrix inversion option of the DDA code described in Evans and Stephens (1995a) is used for particles with 5000 or fewer dipoles. For larger particles (and all snowflake aggregates) the ADDA code of Yurkin and Hoekstra (2011) is run, which uses the FFT conjugate-gradient solution method and can be run on parallel processors. A least squares fitting procedure is used to convert the phase (Mueller) matrix ADDA output as a function of angle to Legendre series coefficients. The ice indices of refraction are from Matzler (2006). The DDA computations are performed for 18 hexagonal plates, 40 plate aggregates, 36 sphere aggregates, and 89 snow aggregates.

The scattering tables contain the extinction, single scattering albedo, and Legendre coefficients of the phase function for gamma size distributions (in $D_{\mathrm{e}}$ ) of randomly oriented particles. The scattering properties are tabulated as a function of $D_{\mathrm{me}}, D_{\mathrm{e}}$ dispersion, particle shape, and temperature. The range of $D_{\text {me }}$ available in a scattering table depends on the particle shape (see Table B1), mainly due to choices about what sizes are appropriate for each shape. There are usually two particle shapes available at each $D_{\text {me }}$, so that there is uncertainty due to particle shape. The $D_{\text {me }}$ are always spaced at $0.5 \mathrm{~dB}$ intervals. Four $D_{\mathrm{e}}$ dispersions (size distribution widths) are tabulated in the scattering tables: at $0.1,0.3,0.5$, and 0.7. The temperature dependence of the ice scattering properties is represented with two temperatures: $215 \mathrm{~K}$ and $260 \mathrm{~K}$. The real part of the index of refraction of ice at submillimeter wavelengths varies linearly in temperature. The imaginary index actually varies nearly quadratically in temperature, but, since the imaginary part is small, the error in scattering properties is deemed not significant using these two well-placed temperatures. Using two temperatures decreases the scattering computations and the interpolation time.

\section{B4 Melting model}

The lower frequencies of CoSSIR, such as $220 \mathrm{GHz}$ and $183.3 \pm 6.6 \mathrm{GHz}$, have substantial sensitivity to hydrometeors below the melting level, though have little sensitivity to the surface or boundary layer in tropical atmospheres. Therefore, an ice cloud retrieval algorithm for CoSSIR, and millimeter-wave radiometers in general, needs to model melting ice, raindrops, and cloud droplets below the melting level. The approach taken here is to use a simple melting model of the ice particles, which is appropriate for stratiform regions (small updrafts). This melting model is implemented in the scattering tables, in which the scattering properties are a function of temperature, rather than explicitly in the $\mathrm{CDF} / \mathrm{EOF}$ generation program or retrieval program.

The melting model operates on single particles, and uses a fall-speed relation and a heat transfer parameterization to calculate the melted mass fraction as a function of height, and thus temperature using a specified lapse rate. The diameter of the initial ice particle is taken to be the area equivalent diameter. The particles are assumed to be spherical, and the fall velocity is obtained from Heymsfield and Westbrook (2010). The particle mass that melts as it falls a distance $\mathrm{d} z$ is from Eq. (1) in Bauer et al. (2000), which is derived from a Rutledge and Hobbs (1983) parameterization that includes ventilation. The diameter $D_{\mathrm{m}}$ of the melting particle is obtained from

$D_{\mathrm{m}}^{3}=f_{\mathrm{m}} D_{\mathrm{r}}^{3}+\left(1-f_{\mathrm{m}}\right) D_{\mathrm{i}}^{3}$,

where $f_{\mathrm{m}}$ is the melted mass fraction, $D_{\mathrm{i}}$ the equivalent area diameter of the original ice particle, and $D_{\mathrm{r}}$ the diameter of the fully melted raindrop (from the ice particle mass). This melting model ignores collection, evaporation, and liquid shedding, and assumes that the updraft velocity is negligible. 
Table B1. Range of ice water content weighted equivalent sphere diameter.

\begin{tabular}{lrrc}
\hline Particle shape & Min $D_{\text {me }}$ & Max $D_{\text {me }}$ & Number $D_{\text {me }}$ \\
\hline Plate aggregates & 6.310 & 398.1 & 37 \\
Sphere aggregates & 5.012 & 1584.9 & 51 \\
Snow aggregates & 63.10 & 1584.9 & 29 \\
Hail (solid spheres) & 398.1 & 3162.3 & 19 \\
\hline
\end{tabular}

The microwave scattering properties of the melting particles are calculated for spheroids using the T-matrix method code of Mishchenko (Mishchenko and Travis, 1998). Since assuming spheroids and a dielectric mixing rule is a poor approximation to the microwave scattering properties of realistic large ice particles, a radiatively equivalent sphere or spheroid is found to match the DDA ice scattering properties of the ice particle. First the DDA ice scattering properties are extrapolated in temperature to $273.2 \mathrm{~K}$. An attempt is made to match three scattering quantities with Mie theory for spheres by adjusting the "scattering" radius and the real and imaginary parts of the index of refraction of the ice/air mixture. For radar backscattering (i.e., $94 \mathrm{GHz}$ ) the fractional difference in backscatter and extinction and the difference in single scattering albedo are minimized. For the radiometer channels, the fractional difference in extinction and the difference in single scattering albedo and asymmetry parameter are minimized. In both cases the downhill simplex simulated annealing method is used to minimize an objective function that combines the three scattering quantities. Usually a perfect match to the three DDA scattering quantities can be found using the radiatively equivalent sphere concept. If an excellent sphere match is not found, then the T-matrix method is used to find a matching spheroid by adjusting the equivalent radius, aspect ratio, and index of refraction. When the scattering properties of the melting particles are calculated with the T-matrix method, it is the DDA matching ice spheroid properties (radius, aspect ratio, and index of refraction) that are mixed with the water properties. The dielectric constant of the melting particle is calculated with the Maxwell Garnett formula mixing for ice/air inclusions in water, and the index of refraction of water is from Ray (1972). The particle temperature is at the melting point until the particle is fully melted, and then the ambient temperature is assumed. The T-matrix radius is obtained by interpolating between the cubes of the raindrop radius and the DDA matching radius using the melted mass fraction $f_{\mathrm{m}}$. The aspect ratio is found by linearly interpolation with $f_{\mathrm{m}}$. When the DDA properties are matched by Mie theory, then the aspect ratio of the melting particle remains at 1 . After the particle is fully melted, a spherical shape is assumed and the T-matrix calculations are done for liquid water for the rest of the profile.

Once the scattering properties of the single melting and melted particles are calculated, the scattering properties over gamma size distributions are assembled into scattering tables. The structure of the melting particle scattering tables must be the same as that of the ice particles, except for the different temperature range, of course. The scattering properties of the melting/melted particles are calculated at 20 temperatures ranging from 272 to $300 \mathrm{~K}(0.5 \mathrm{~K}$ spacing from 273 to $277 \mathrm{~K}, 1.0 \mathrm{~K}$ spacing from 277 to $282 \mathrm{~K}$, and $2.0 \mathrm{~K}$ spacing from 282 to $290 \mathrm{~K}$ ).

\section{Appendix C}

\section{Stochastic generation of radar reflectivity}

CloudSat radar reflectivity is randomly simulated for layers in which the reflectivity is below the $-26 \mathrm{dBZ}$ threshold, but are known to be cloudy from the CALIPSO lidar cloud mask (averaged to the CloudSat range bins in the CloudSat GEOPROF-LIDAR product as described in Mace et al., 2009). The procedure samples stochastically from a Bayesian posterior probability distribution function (pdf). The prior pdf has three factors, which result in appropriate horizontal and vertical smoothness and agreement with the height-dependent reflectivity distribution obtained from the input ice microphysical statistics. The likelihood function in the Bayesian analysis assures agreement with the CloudSat reflectivity and the reflectivity noise distribution.

The prior pdf for the simulated reflectivity is the product of three Gaussian pdfs in dBZ. The first Gaussian prior distribution is for the difference in reflectivity between layers at the same altitude in adjacent CloudSat columns and assures horizontal smoothness. This pdf has zero mean and standard deviation calculated from the reflectivity difference of layers in adjacent columns. The second Gaussian prior distribution is for the difference in reflectivity between vertically adjacent layers in the same column and assures vertical smoothness and extrapolation to lower reflectivities with height. The mean and standard deviation of this pdf are calculated from adjacent pairs of layers in which the upper layer has reflectivity less than that of the lower layer. The statistics for these two Gaussian prior pdfs are only accumulated for layers in which the reflectivity is between the specified threshold (e.g., $-26 \mathrm{dBZ}$ ) and $5 \mathrm{dBZ}$ greater. The third Gaussian prior pdf is determined from the reflectivity distribution calculated for each hydrometeor layer height from the input (temperature dependent) ice microphysical statistics. For the tropical examples shown here, the ice microphysical pdfs have standard deviations around $10 \mathrm{dBZ}$ and means that decrease from $-17.4 \mathrm{dBZ}$ for $10.0-10.5 \mathrm{~km}$ to $-36.2 \mathrm{dBZ}$ for 14.5 $15.0 \mathrm{~km}$. The horizontal smoothness pdf standard deviation is $2.9 \mathrm{dBZ}$, and the vertical pdf mean and standard deviation are $-5.4 \mathrm{dBZ}$ and $3.7 \mathrm{dBZ}$.

The Bayesian likelihood pdf for the simulated reflectivity is a Gaussian in reflectivity factor $Z$ (units of $\left.\mathrm{mm}^{6} \mathrm{~m}^{-3}\right)$ around the CloudSat measured reflectivity $\left(Z_{\mathrm{obs}}\right)$. 
The Gaussian likelihood pdf standard deviation $\left(\sigma_{Z}\right)$ is the CloudSat range bin noise rms divided by the square root of the number of CloudSat range bins that fit into the layer thickness. The CloudSat reflectivity noise rms is determined from valid $(Z>-88.8 \mathrm{dBZ})$ stratospheric range bins in the target region separately for each orbit. For the tropical CloudSat columns used here, the mean noise rms is $9.48 \times 10^{-4} \mathrm{~mm}^{6} \mathrm{~m}^{-3}(-30.2 \mathrm{dBZ})$ and there is only about a $1 \%$ variation among different orbits. The Bayesian posterior pdf is stochastically sampled by generating samples from the Gaussian prior pdf in $\mathrm{dBZ}$, converting to $Z$, and using the rejection method to sample the likelihood function (with $\left.p=\exp \left[-\left(Z-Z_{\mathrm{obs}}\right)^{2} / \sigma_{Z}^{2}\right]\right)$. Radar reflectivity is simulated for layers with lidar cloud fraction greater than 0.5 for layers completely above the freezing level. Since the stochastically simulated reflectivity depends on neighboring values, the sweeping order is first in columns and then in layers from bottom to top. Below the ice region the CloudSat measured reflectivity is used if it is above a threshold, though the threshold depends on whether the lidar detects cloud. Figure 2 shows an example of the radar reflectivity profiles with and without the simulated reflectivity.

Acknowledgements. The authors thank Bryan Monosmith for engineering support of CoSSIR in the lab and during TC4. Glenn Diskin and James Podolske provided the DLH data and Robert Herman provided the JLH data to the ESPO archive. The NASA CloudSat project provided the CloudSat GEOPROF, GEOPROF-LIDAR, ECMWF-AUX files through the CloudSat Data Processing Center. Two anonymous reviewers are thanked for helping to improve the clarity of this article. KFE was supported in this work by NASA Award NNX07AK83G.

Edited by: S. Schmidt

\section{References}

Andrieu, C. and Thoms, J.: A tutorial on adaptive MCMC, Stat. Comput., 18, 343-373, 2008.

Auer, A. H. and Veal, D. L.: The dimension of ice crystals in natural clouds, J. Atmos. Sci., 27, 919-926, 1970.

Austin, R. T., Heymsfield, A. J., and Stephens, G. L.: Retrieval of ice cloud microphysical parameters using the CloudSat millimeter-wave radar and temperature, J. Geophys. Res., 114, D00A23, doi:10.1029/2008JD010049, 2009.

Bannister, R. N.: A review of forecast error covariance statistics in atmospheric variational data assimilation, II: Modelling the forecast error covariance statistics, Q. J. Roy. Meteorol. Soc., 134, 1971-1996, 2008.

Battaglia, A., Augustynek, T., Tanelli, S., and Kollias, P.: Multiple scattering identification in spaceborne W-band radar measurements of deep convective cores, J. Geophys. Res., 116, D19201, doi:10.1029/2011JD016142, 2011.

Bauer, P., Khain, A., Pokrovsky, A., Meneghini, R., Kummerow, C., Marzano, F., and Poieres Baptista, J. P. V.: Combined cloud microwave radiative transfer modeling of stratiform rainfall, J. Atmos. Sci., 57, 1082-1104, 2000.

Baumgardner, D., Jonsson, H., Dawson, W., O’Connor, D., and Newton, R.: The cloud, aerosol and precipitation spectrometer: A new instrument for cloud investigations, Atmos. Res., 59-60, 251-264, 2001.

Buehler, S. A., Jimenez, C., Evans, K. F., Eriksson, P., Rydberg, B., Heymsfield, A. J., Stubenrauch, C. J., Lohmann, U., Emde, C., John, V. O., Sreerekha, T. R., and Davis, C. P.: A concept for a satellite mission to measure cloud ice water path, ice particle size, and cloud altitude, Q. J. Roy. Meteorol. Soc., 133, 109-128, 2007.

Clough, S. A., Shephard, M. W., Mlawer, E. J., Delamere, J. S., Iacono, M. J., Cady-Pereira, K., Boukabara, S., and Brown, P. D.: Atmospheric radiative transfer modeling: a summary of the AER codes, Short Communication, J. Quant. Spectrosc. Ra., 91, 233-244, 2005.

Cooper, S. J., L'Ecuyer, T., Gabriel, P., Baran, A. J., and Stephens, G. L.: Objective assessment of the information content of visible and infrared radiance measurements for cloud microphysical property retrievals over the global oceans, Part II: Ice clouds, J. Appl. Meteorol. Clim., 45, 42-62, 2006.

Defer, E., Prigent, C., Aires, F., Pardo, J. R., Walden, C. J., Zanife, O. Z., Chaboureau, J. P., and Pinty, J. P.: Development of precipitation retrievals at millimeter and sub-millimeter wavelengths for geostationary satellites, J. Geophys. Res., 113, D08111, doi:10.1029/2007JD008673, 2008.

Delanoe, J. and Hogan, R. J.: Combined CloudSat-CALIPSOMODIS retrievals of the properties of ice clouds, J. Geophys. Res., 115, D00H29, doi:10.1029/2009JD012346, 2010.

Diskin, G. S., Sachse, G. W., Podolske, J. R., and Slate, T. A.: Openpath airborne tunable diode laser hygrometer, Proc. SPIE, 196, 4817, doi:10.1117/12.453736, 2002.

Eliasson, S., Buehler, S. A., Milz, M., Eriksson, P., and John, V. O.: Assessing observed and modelled spatial distributions of ice water path using satellite data, Atmos. Chem. Phys., 11, 375391, doi:10.5194/acp-11-375-2011, 2011.

Evans, K. F.: SHDOMPPDA: A radiative transfer model for cloudy sky data assimilation, J. Atmos. Sci., 64, 3858-3868, 2007.

Evans, K. F. and Stephens, G. L.: Microwave radiative transfer through clouds composed of realistically shaped ice crystals. Part I: Single scattering properties, J. Atmos. Sci., 52, 2041-2057, 1995a.

Evans, K. F. and Stephens, G. L.: Microwave radiative transfer through clouds composed of realistically shaped ice crystals, Part II: Remote sensing of ice clouds, J. Atmos. Sci., 52, 20582072, 1995b.

Evans, K. F., Walter, S. J., Heymsfield, A. J., and Deeter, M. N.: Modeling of submillimeter passive remote sensing of cirrus clouds, J. Appl. Meteorol., 37, 184-205, 1998.

Evans, K. F., Walter, S. J., Heymsfield, A. J., and McFarquhar, G. M.: The Submillimeter-wave cloud ice radiometer: Simulations of retrieval algorithm performance, J. Geophys. Res., 107, 4028, doi:10.1029/2001JD000709, 2002.

Evans, K. F., Wang, J. R., Racette, P. E., Heymsfield, G., and Li, L.: Ice cloud retrievals and analysis with data from the Compact Scanning Submillimeter Imaging Radiometer and the Cloud Radar System during CRYSTAL-FACE, J. Appl. Meteorol., 44, 839-859, 2005. 
Ferraro, R. R., Weng, F., Grody, N. C., Zhao, L., Meng, H., Kongoli, C., Pellegrino, P., Qiu, S., and Dean, C.: NOAA operational hydrological products derived from the advanced microwave sounding unit, IEEE T. Geosci. Remote, 43, 1036-1049, 2005.

Gasiewski, A. J.: Numerical sensitivity analysis of passive EHF and SMMW channels to tropospheric water vapor, clouds, and precipitation, IEEE T. Geosci. Remote, 30, 859-870, 1992.

Gillespie, D. T.: An exact method for numerically simulating the stochastic coalescence process in a cloud, J. Atmos. Sci., 32, 1977-1989, 1975.

Gravner, J. and Griffeath, D.: Modeling snow crystal growth II: A mesoscopic lattice map with plausible dynamics, available at: http://psoup.math.wisc.edu/Snowfakes.htm, Physica D: Nonlinear Phenomena, 237, 385-404, 2008.

Heymsfield, A. J. and Westbrook, C. D.: Advances in the estimation of ice particle fall speeds using laboratory and field measurements, J. Atmos. Sci., 67, 2469-2482, 2010.

Jensen, E. J., Lawson, P., Baker, B., Pilson, B., Mo, Q., Heymsfield, A. J., Bansemer, A., Bui, T. P., McGill, M., Hlavka, D., Heymsfield, G., Platnick, S., Arnold, G. T., and Tanelli, S.: On the importance of small ice crystals in tropical anvil cirrus, Atmos. Chem. Phys., 9, 5519-5537, doi:10.5194/acp-9-5519-2009, 2009.

Jimenez, C., Buehler, S. A., Rydberg, B., Eriksson, P., and Evans, K. F.: Performance simulations for a submillimetre wave cloud ice satellite instrument, Q. J. Roy. Meteorol. Soc., 133, 129-149, doi:10.1002/qj.134, 2007.

King, M. D., Tsay, S.-C., Platnick, S. E., Wang, M., and Liou, K.N.: Cloud retrieval algorithms for MODIS: Optical thickness, effective particle radius, and thermodynamic phase, Tech. rep., MODIS Science Team, MODIS Algorithm Theoretical Basis Document No. ATBD-MOD-05, NASA Goddard Space Flight Center, Greenbelt, MD USA, 1997.

Kulie, M. S., Bennartz, R., Greenwald, T. J., Chen, Y., and Weng, F.: Uncertainties in microwave properties of frozen precipitation: Implications for remote sensing and data assimilation, J. Atmos. Sci., 67, 3471-3487, 2010.

Lawson, R. P., O'Connor, D., Zmarzly, P., Weaver, K., Baker, B., Mo, Q., and Jonsson, H.: The 2D-S (Stereo) probe: Design and preliminary tests of a new airborne, high-speed, high-resolution particle imaging probe, J. Atmos. Ocean. Tech., 23, 1462-1477, 2006.

Li, L., Heymsfield, G. M., Racette, P. E., Tian, L., and Zenker, E.: A 94-GHz Cloud Radar System on a NASA High-Altitude ER-2 Aircraft, J. Atmos. Ocean. Tech., 21, 1378-1388, 2004.

Mace, G. G., Zhang, Q., Vaughan, M., Marchand, R., Stephens, G., Trepte, C., and Winker, D.: A description of hydrometeor layer occurrence statistics derived from the first year of merged Cloudsat and CALIPSO data, J. Geophys. Res., 114, D00A26, doi:10.1029/2007JD009755, 2009.

Marchand, R. T., Mace, G. G., and Ackerman, T. P.: Hydrometeor detection using CloudSat: An Earth orbiting $94 \mathrm{GHz}$ cloud radar, J. Atmos. Ocean. Tech., 25, 519-533, doi:10.1175/2007JTECHA1006.1, 2008.

Maruyama, K. and Fujiyoshi, Y.: Monte Carlo Simulation of the Formation of Snowflakes, J. Atmos. Sci., 62, 1529-1544, 2005.

Matzler, C.: Microwave dielectric properties of ice, in: Thermal Microwave Radiation, Applications for Remote Sensing, edited by: Matzler, C., Rosenkranz, P. W., Battaglia, A., and Wigneron, J.
P., IET Electromagnetic Waves Series, Vol. 52, Institute of Engineering and Technology, Stevenage, UK, 2006.

Mishchenko, M. I. and Travis, L. D.: Capabilities and limitations of a current FORTRAN implementation of the T-matrix method for randomly oriented, rotationally symmetric scatterers, J. Quant. Spectrosc. Ra., 60, 309-324, 1998.

Petty, G. W. and Huang, W.: Microwave backscatter and extinction by soft ice spheres and complex snow aggregates, J. Atmos. Sci., 67, 769-787, 2010.

Posselt, D., L'Ecuyer, T. S., and Stephens, G. L.: Exploring the error characteristics of thin ice cloud property retrievals using a Markov chain Monte Carlo algorithm, J. Geophys. Res., 113, D24206, doi:10.1029/2008JD010832, 2008.

Ray, P. S.: Broadband complex refractive indices of ice and water, Appl. Optics, 11, 1836-1844, 1972.

Rodgers, C. D.: Inverse Methods for Atmospheric Sounding: Theory and Practice, World Sci., River Edge, NJ, 238 pp., 2000.

Rossow, W. B. and Schiffer, R. A.: Advances in understanding clouds from ISCCP, B. Am. Meteorol. Soc., 80, 2261-2288, 1999.

Rutledge, S. A. and Hobbs, P. V.: The mesoscale and microscale structure and organization of clouds and precipitation in midlatitude cyclones, VIII: A model for the "seeder-feeder" process in warm-frontal rainbands, J. Atmos. Sci., 40, 1185-1206, 1983.

Rydberg, B., Eriksson, P., Buehler, S. A., and Murtagh, D. P.: NonGaussian Bayesian retrieval of tropical upper tropospheric cloud ice and water vapour from Odin-SMR measurements, Atmos. Meas. Tech., 2, 621-637, doi:10.5194/amt-2-621-2009, 2009.

Seo, E. and Liu, G.: Retrievals of cloud ice water path by combining ground cloud radar and satellite high-frequency microwave measurements near the ARM SGP site, J. Geophys. Res., 110, D14203, doi:10.1029/2004JD005727, 2005.

Stephens, G. L., Vane, D. G., Tanelli, S., Im, E., Durden, S., Rokey, M., Reinke, D., Partain, P., Mace, G. G., Austin, R., L'Ecuyer, Haynes, T. J., Lebsock, M., Suzuki, K., Waliser, D., Wu, D., Kay, J., Gettelman, A., Wang, Z., and Marchand, R.: CloudSat mission: Performance and early science after the first year of operation, J. Geophys. Res., 113, D00A18, doi:10.1029/2008JD009982, 2008.

Tamminen, J. and Kyrola, E.: Bayesian solution for nonlinear and non-Gaussian inverse problems by Markov chain Monte Carlo method, J. Geophys. Res., 106, 14377-14390, 2001

Toon, O. B., Starr, D. O., Jensen, E. J., Newman, P. A., Platnick, S., Schoeberl, M. R., Wennberg, P. O., Wofsy, S. C., Kurylo, M. J., Maring, H., Jucks, K. W., Craig, M. S., Vasques, M. F., Pfister, L., Rosenlof, K. H., Selkirk, H. B., Colarco, P. R., Kawa, S. R., Mace, G. G., Minnis, P., and Pickering, K. E.: Planning, implementation, and first results of the Tropical Composition, Cloud and Climate Coupling Experiment (TC4), J. Geophys. Res., 115, D00J04, doi:10.1029/2009JD013073, 2010.

Venema, V., Ament, F., and Simmer, C.: A Stochastic Iterative Amplitude Adjusted Fourier Transform algorithm with improved accuracy, Nonlin. Processes Geophys., 13, 321-328, doi:10.5194/npg-13-321-2006, 2006.

Wu, D. L., Jiang, J. H., and Davis, C. P.: EOS MLS cloud ice measurements and cloudy-sky radiative transfer model, IEEE T. Geosci. Remote, 44, 1156-1165, doi:10.1109/TGRS.2006.869994, 2006. 
Wu, D. L., Austin, R. T., Deng, M., Durden, S. L., Heymsfield, A. J., Jiang, J. H., Lambert, A., Li, J.-L., Livesey, N. J., McFarquhar, G. M., Pittman, J. V., Stephens, G. L., Tanelli, S., Vane, D. G., and Waliser, D. E.: Comparisons of global cloud ice from MLS, CloudSat, and correlative data sets, J. Geophys. Res., 114, D00A24, doi:10.1029/2008JD009946, 2009.
Yurkin, M. A. and Hoekstra, A. G.: The discrete-dipoleapproximation code ADDA: Capabilities and known limitations, J. Quant. Spectrosc. Ra., 112, 2234-2247, 2011.

Zhang, Y. and Mace, G. G.: Retrieval of cirrus microphysical properties with a suite of algorithms for airborne and spaceborne lidar, radar, and radiometer data, J. Appl. Meteorol. Clim., 45, 1665-1689, 2006. 